\title{
A novel high-order algorithm for the numerical estimation of fractional differential equations
}

\author{
Mohammad Shahbazi Asl ${ }^{1}$, Mohammad Javidi, ${ }^{1, *}$, Yubin Yan ${ }^{2}$ \\ ${ }^{1}$ Department of Applied Mathematics, University of Tabriz, Tabriz, Iran \\ ${ }^{2} 2$ Department of Mathematics, University of Chester, Chester CHI 4BJ, UK
}

\begin{abstract}
This paper uses polynomial interpolation to design a novel high-order algorithm for the numerical estimation of fractional differential equations. The Riemann-Liouville fractional derivative is expressed by using the Hadamard finite-part integral and the piecewise cubic interpolation polynomial is utilized to approximate the integral. The detailed error analysis is presented an it is established that the convergence order of the algorithm is $O\left(h^{4-\alpha}\right)$. Asymptotic expansion for the error of presented algorithm is also investigated. Some numerical examples are provided and compared with the exact solution to show that the numerical results are in well agreement with the theoretical ones and also to illustrate the accuracy and efficiency of the proposed algorithm.

Keywords: Fractional differential equation, Caputo fractional derivative,
\end{abstract}

Riemann-Liouville fractional derivative, Error estimates, Hadamard finite-part integral

2010 MSC: 26A33, 65L70, 65L05

\section{Introduction}

The beginning of the fractional calculus dates back to the end of the 17th century. However, until recently, due to its complexity and the lack of application background it has been investigated mainly from a mathematical point of view [1, 2]. In the 19th 5 century, a complete theory suitable for modern mathematical developments has been

\footnotetext{
* Corresponding author

Email address: mo_javidi@tabrizu.ac.ir (Mohammad Javidi)
} 
formalized by mathematicians [3]. Nowadays, fractional calculus is a wellestablished theory, which is widely applied in many fields of science, engineering, and mathematics [4, 5].

The most important advantage of using fractional order differential equation instead of integer-order one is that it is nonlocal in nature [6, 7]]. In other words, the fractional calculus provides an excellent instrument for the description of memory and hereditary properties of many physical phenomena and processes [8, 9]. With this advantage, fractional order models are more realistic and practical than the classical integer-order models [10, 11]. On the other hand, it is difficult to formulate an accurate and fast numerical method due to this trait [12]. In general, the exact solution of most fractional differential equations is very difficult even impossible to obtain [13, 14, 15]. Even though for linear fractional differential equations with constant coefficients, analytical solutions are available by utilizing Laplace-Fourier transform techniques, but these solutions always contain some infinite series such as Mittag-Leffler function which make evaluation very expensive $[16,17]$. For this reason, approximate and numerical techniques are playing an important role in identifying the solution behavior of such fractional equations and exploring their applications [18].

This paper is concerned with design a novel high-order algorithm for the numerical estimation of fractional differential equations of the general form:

$$
\left\{\begin{array}{l}
{ }_{0}^{C} D_{t}^{\alpha} y(t)=\mu y(t)+g(t), \quad t \in[0,1], \\
y\left(t_{0}\right)=y_{0}, \quad,
\end{array}\right.
$$

where ${ }_{0}^{C} D_{t}^{\alpha} y(t)$ denotes the Caputo fractional order derivative, $0<\alpha<1, \mu<0$ is a constant and $g$ is a given function. In pure mathematical viewpoint, Riemann-Liouville derivative is more welcome than Caputo derivative but this is not always the most convenient definition for real applications [19]. However, this definition is less popular in real applications because of the fact that it requires initial conditions to be expressed in terms of fractional integrals and their derivatives, which there is no known physical interpolation for such types of initial conditions [20, 21]. The use of Caputo derivative 30 in Eq. (1) is partly because of the convenience to specify the initial conditions. Since Caputos fractional derivative allows one to couple the fractional differential equations 
with initial conditions in the traditional form, namely, the initial conditions are expressed in terms of values of the unknown function and its integer order derivatives which have clear physical meaning [22, 23].

In the last decades, there has been a growing interest to design numerical methods to resolve (1). Diethelm et al. proposed predictor-corrector method to solve FDEs in [24] and give the corresponding detailed error analysis in [25]. Shahbazi Asl and Javidi utilized 3/8 Simpson's rule to improve the accuracy of the predictor-corrector method and present error and stability analysis of the method. Li et al. designed a proposed the rectangle formula and trapezoid formula based on the non-uniform meshes to construct finite difference methods for solving FDEs [26].

Diethelm constructed a numerical method with the convergence order $O\left(h^{2-\alpha}\right)$ for solving linear FDEs [27]. The fractional Riemann-Liouville derivative is composed by using the Hadamard finite-part integral in this research and the piecewise linear interpolation polynomial is utilized to approximate the integral. This method is applied to design numerical methods for solving some fractional partial differential equations [28, 29]. Yan et al. modified the method in [27] by using the piecewise quadratic interpolation polynomial [30]. The convergence order of this method was proved to be $O\left(h^{3-\alpha}\right)$. The same authors applied this method to design numerical method for solving time-space-fractional partial differential equations [31]. Pal et al. utilized the method of [30] to design an extrapolation algorithm for solving FDEs [32]. The authors of [33] proved the error estimates of the methods in [27] and [30].

The purpose of this paper is to extend the numerical method of [30] for solving Eq. (1) by using the piecewise cubic interpolation polynomial. The advantage of the new modified method is that first, the presented numerical algorithm has a higher order convergence order $\left(O\left(h^{4-\alpha}\right)\right)$. Second, the numerical estimation of the first integrals and by a similar way the starting values with acceptable accuracy is presented. Finally, the proofs of the truncation error estimates are given. 


\section{Numerical method}

\subsection{Extending Yan's methos}

This section present a novel high order numerical algorithm for solving (1). It is worth pointing out that, any closed interval $[0, T]$ can be transformed to the interval $[0,1]$, so this choice does not made essential restriction. For $0<\alpha<1$, Eq. (1) can be written as

$$
{ }_{0}^{R} D_{t}^{\alpha}\left(y(t)-y_{0}\right)=\mu y(t)+g(t), \quad t \in[0,1],
$$

where ${ }_{0}^{R} D_{t}^{\alpha} y(t)$ denotes the Riemann-Liouville fractional derivative defined by [34, 28]

$$
{ }_{0}^{R} D_{t}^{\alpha} y(t)=\frac{1}{\Gamma(-\alpha)} \oint_{0}^{t}(t-\tau)^{-1-\alpha} y(\tau) d \tau
$$

in which the integral $\oint_{0}^{t}(t-\tau)^{-1-\alpha} y(\tau) d \tau$, denotes the Hadamard finite-part integral. Recall that the Riemann-Liouville fractional derivative of a constant $y_{0}$ is [35]:

$$
{ }_{0}^{R} D_{t}^{\alpha} y_{0}=\frac{y_{0}}{\Gamma(1-\alpha)} t^{-\alpha}
$$

To construct the high order scheme, the third-degree compound quadrature formula is used, taken with respect to the weight function $(t-\cdot)^{-1-\alpha}$ to replace the integral naturally. For a fixed positive integer $m$, we introduce an equispaced grid $t_{l}=l h$, $l=0,1,2, \ldots, 3 m$ on the interval $[0,1]$ where $h=\frac{1}{3 m}$ is the step size. Throughout this paper set $y_{l}$ be the numerical approximation of $y\left(t_{l}\right)$. The novel algorithm will be designed by three steps.

Step 1 At the node $t_{l}=\frac{l}{3 m}$, with $l=3 j, j=1,2, \ldots, m$ :

Utilizing Eqs. (3) and (4) the Eq. 2] satisfies

$$
\mu y\left(t_{3 j}\right)=\frac{1}{\Gamma(-\alpha)} \oint_{0}^{t_{3 j}}\left(t_{3 j}-\tau\right)^{-1-\alpha} y(\tau) d \tau-\frac{y_{0}}{\Gamma(1-\alpha)} t_{3 j}^{-\alpha}-g\left(t_{3 j}\right) .
$$

Now the integral in (5) is approximated by the following procedure.

$$
\oint_{0}^{t_{3 j}}\left(t_{3 j}-\tau\right)^{-1-\alpha} y(\tau) d \tau=t_{3 j}^{-\alpha} \oint_{0}^{1} u^{-1-\alpha} y\left(t_{3 j}-t_{3 j} u\right) d u=t_{3 j}^{-\alpha} \oint_{0}^{1} u^{-1-\alpha} f(u) d u
$$


where $f(u)=y\left(t_{3 j}-t_{3 j} u\right)$. Set $f_{u}=y_{3 j-3 j \cdot u}$ be the numerical approximation of $f(u)$. The piecewise cubic interpolation polynomial is utilized to approximate $f(u)$ in a such a way that:

$$
\oint_{0}^{1} u^{-1-\alpha} f(u) d u=\oint_{0}^{3 j} u^{-1-\alpha} P_{3}(u) d u+R_{3 j}(f) \approx I^{3 j},
$$

where $R_{3 j+1}(f)$ is the reminder term. This integral $\left(I^{l}, l=3 j\right)$ can be computed numerically as follows:

$$
\begin{aligned}
I^{l} & =\oint_{0}^{\frac{3 j}{T}} u^{-1-\alpha} P_{3}(u) d u=\left[\oint_{0}^{\frac{3}{T}}+\sum_{k=2}^{j} \int_{\frac{3 k-3}{l}}^{\frac{3 k}{T}}\right] u^{-1-\alpha} P_{3}(u) d u \\
& =\oint_{0}^{\frac{3}{T}} u^{-1-\alpha}\left[l_{0, l}^{1}(u) f_{0}+l_{1, l}^{1}(u) f_{\frac{1}{T}}+l_{2, l}^{1}(u) f_{\frac{2}{T}}+l_{3, l}^{1}(u) f_{\frac{3}{T}}\right] d u \\
& +\sum_{k=2}^{j} \int_{\frac{3 k-3}{l}}^{\frac{3 k}{T}} u^{-1-\alpha}\left[l_{3 k-3, l}^{k}(u) f_{\frac{3 k-3}{l}}+l_{3 k-2, l}^{k}(u) f_{\frac{3 k-2}{l}}+l_{3 k-1, l}^{k}(u) f_{\frac{3 k-1}{l}}+l_{3 k, l}^{k}(u) f_{\frac{3 k}{l}}\right] d u \\
& I^{l} \approx \sum_{k=1}^{j}\left[W_{3 k-3, l}^{k} f_{\frac{3 k-3}{l}}+W_{3 k-2, l}^{k} f_{\frac{3 k-2}{l}}+W_{3 k-1, l}^{k} f_{\frac{3 k-1}{l}}+W_{3 k, l}^{k} f_{\frac{3 k}{T}}\right] \\
& =\sum_{k=1}^{j}\left[W_{3 k-3, l}^{k} y_{l-(3 k-3)}+W_{3 k-2, l}^{k} y_{l-(3 k-2)}+W_{3 k-1, l}^{k} y_{l-(3 k-1)}+W_{3 k, l}^{k} y_{l-3 k}\right]
\end{aligned}
$$

in which $l_{i, l}^{k}(u)$ are the cubic Lagrange polynomials and we have for $i=3 k-3,3 k-$ $2,3 k-1,3 k$,

$$
\begin{gathered}
W_{i, l}^{1}=\oint_{0}^{\frac{3}{T}} u^{-1-\alpha} l_{i, l}^{1}(u) d u, \quad W_{i, l}^{k}=\int_{\frac{3 k-3}{l}}^{\frac{3 k}{l}} u^{-1-\alpha} l_{i, l}^{k}(u), \text { for } k=2, \ldots, j, \\
l_{3 k-3, l}^{k}=\frac{\left(u-\frac{3 k-2}{l}\right)}{\left(\frac{3 k-3}{l}-\frac{3 k-2}{l}\right)} \frac{\left(u-\frac{3 k-1}{l}\right)}{\left(\frac{3 k-3}{l}-\frac{3 k-1}{l}\right)} \frac{\left(u-\frac{3 k}{l}\right)}{\left(\frac{3 k-3}{l}-\frac{3 k}{l}\right)}, \\
l_{3 k-2, l}^{k}=\frac{\left(u-\frac{3 k-3}{l}\right)}{\left(\frac{3 k-2}{l}-\frac{3 k-3}{l}\right)} \frac{\left(u-\frac{3 k-1}{l}\right)}{\left(\frac{3 k-2}{l}-\frac{3 k-1}{l}\right)} \frac{\left(u-\frac{3 k}{l}\right)}{\left(\frac{3 k-2}{l}-\frac{3 k}{l}\right)}, \\
l_{3 k-1, l}^{k}=\frac{\left(u-\frac{3 k-3}{l}\right)}{\left(\frac{3 k-1}{l}-\frac{3 k-3}{l}\right)} \frac{\left(u-\frac{3 k-2}{l}\right)}{\left(\frac{3 k-1}{l}-\frac{3 k-2}{l}\right)} \frac{\left(u-\frac{3 k}{l}\right)}{\left(\frac{3 k-1}{l}-\frac{3 k}{l}\right)}, \\
l_{3 k, l}^{k}=\frac{\left(u-\frac{3 k-3}{l}\right)}{\left(\frac{3 k}{l}-\frac{3 k-3}{l}\right)} \frac{\left(u-\frac{3 k-2}{l}\right)}{\left(\frac{3 k}{l}-\frac{3 k-2}{l}\right)} \frac{\left(u-\frac{3 k-1}{l}\right)}{\left(\frac{3 k-1}{l}-\frac{3 k-1}{l}\right)}
\end{gathered}
$$


In this way, one can design the following numerical algorithm to approximate $y\left(t_{3 j}\right)$, $j=1,2, \ldots, m$

$$
\begin{aligned}
\mu y_{3 j}= & \frac{t_{3 j}^{-\alpha}}{\Gamma(-\alpha)} \sum_{k=1}^{j}\left[W_{3 k-3,3 j}^{k} y_{3 j-(3 k-3)}+W_{3 k-2,3 j}^{k} y_{3 j-(3 k-2)}+W_{3 k-1,3 j}^{k} y_{3 j-(3 k-1)}\right. \\
& \left.+W_{3 k, 3 j}^{k} y_{3 j-3 k}\right]-\frac{y_{0}}{\Gamma(1-\alpha)} t_{3 j}^{-\alpha}-g\left(t_{3 j}\right)
\end{aligned}
$$

Lemma 1. Fix $0<\alpha<1$. Then with $l=3 j, j=1,2, \ldots, m$, the integral in (8) yields

$$
\oint_{0}^{\frac{3 j}{3 j}} u^{-1-\alpha} P_{3}(u) d u=\sum_{k=0}^{3 j} w_{k, 3 j} f\left(\frac{k}{3 j}\right)=\sum_{k=0}^{3 j} w_{k, 3 j} y\left(t_{3 j-k}\right),
$$

in which $w_{k, 3 j}=\left(\frac{3}{3 j}\right)^{-\alpha} \frac{1}{2(-\alpha)(-\alpha+1)(-\alpha+2)(-\alpha+3)} \tilde{w}_{k, 3 j}$ and,

$$
\tilde{w}_{k, 3 j}= \begin{cases}2 \alpha^{2}+8 \alpha+12, & \text { for } k=0, \\ -\alpha(9 \alpha+27), & \text { for } k=1, \\ 18 \alpha^{2}, & \text { for } k=2, \\ \alpha\left(-2 \alpha^{2}+\alpha-3\right)+F_{1}(1), & \text { for } k=3, j>1, \\ F_{2}(l), & \text { for } k=3 l-2, l=2, \ldots, j, \\ F_{3}(l), & \text { for } k=3 l-1, l=2, \ldots, j, \\ F_{0}(l)+F_{1}(l), & \text { for } k=3 l, \quad l=2,3, \ldots, j-1, \\ F_{0}(j), & \text { for } k=3 j,\end{cases}
$$

$$
\begin{aligned}
F_{0}(l)= & l^{-\alpha}\left[-54 l^{3}-36 l^{2}(\alpha-3)-11 l(\alpha-3)(\alpha-2)-2(\alpha-3)(\alpha-2)(\alpha-1)\right] \\
& +2(l-1)^{1-\alpha}\left[27 l^{2}-9 l(\alpha+3)+\alpha(\alpha+4)+6\right], \\
F_{1}(l)= & -l^{-\alpha}\left[54 l^{3}-36 l^{2}(\alpha-3)+11 l(\alpha-3)(\alpha-2)-2(\alpha-3)(\alpha-2)(\alpha-1)\right] \\
& +2(l+1)^{1-\alpha}\left[27 l^{2}+9 l(\alpha+3)+\alpha(\alpha+4)+6\right], \\
F_{2}(l)= & -9 l^{1-\alpha}\left[\alpha^{2}-5 \alpha+18 l^{2}+8 l(\alpha-3)+6\right] \\
& +18(l-1)^{1-\alpha}\left[\alpha^{2}+9 l^{2}-l(5 \alpha+3)\right], \\
F_{3}(l)= & 18 l^{1-\alpha}\left[\alpha^{2}-5 \alpha+9 l^{2}+5 l(\alpha-3)+6\right] \\
& -9(l-1)^{1-\alpha}\left[\alpha(\alpha+3)+18 l^{2}-4 l(2 \alpha+3)\right] .
\end{aligned}
$$


Proof. The piecewise cubic interpolation polynomial with the nodes $0, \frac{1}{3 j}, \frac{2}{3 j}, \ldots, \frac{3 j}{3 j}$ is used to approximate $f(u)$. We have by setting $l=3 j$ in 8

$$
I^{3 j}=\oint_{0}^{\frac{3 j}{3 j}} u^{-1-\alpha} P_{3}(u) d u=\left[\oint_{0}^{\frac{3}{3 j}}+\sum_{k=2}^{j} \int_{\frac{3 k-3}{3 j}}^{\frac{3 k}{3 j}}\right] u^{-1-\alpha} P_{3}(u) d u
$$

By the definition of the Hadamard finite-part integral [28, 36, 30], one can obtain

$$
\begin{aligned}
I=\oint_{0}^{\frac{3}{3 j}} & u^{-1-\alpha} P_{3}(u) d u=\frac{P_{3}(0)\left(\frac{3}{3 j}\right)^{-\alpha}}{-\alpha}+\int_{0}^{\frac{3}{3 j}} u^{-1-\alpha}(I I) d u, \\
I I=\int_{0}^{u} P_{3}^{\prime}(y) d y= & j \int_{0}^{u}\left[\frac{-1}{2}\left(27 j^{2} y^{2}-36 j y+11\right) f(0)+\frac{9}{2}\left(9 j^{2} y^{2}-10 j y+2\right) f\left(\frac{1}{3 j}\right)\right. \\
& \left.-\frac{9}{2}\left(9 j^{2} y^{2}-8 j y+1\right) f\left(\frac{2}{3 j}\right)+\frac{1}{2}\left(27 j^{2} y^{2}-18 j y+2\right) f\left(\frac{3}{3 j}\right)\right] d y \\
= & \frac{j u}{2}\left[\left(-9 j^{2} u^{2}+18 j u-11\right) f(0)+\left(27 j^{2} u^{2}-45 j u+18\right) f\left(\frac{1}{3 j}\right)\right. \\
& \left.+9\left(-3 j^{2} u^{2}+4 j u-1\right) f\left(\frac{2}{3 j}\right)+\left(9 j^{2} u^{2}-9 j u+2\right) f\left(\frac{3}{3 j}\right)\right],
\end{aligned}
$$

substituting (20) into (19) and calculating the integral yields

$$
\begin{aligned}
I= & \frac{f(0)}{(-\alpha) j^{-\alpha}}-\frac{2\left(\alpha^{2}-5 \alpha+15\right) f(0)-9(\alpha+3) f\left(\frac{1}{3 j}\right)+18 \alpha f\left(\frac{2}{3 j}\right)+\left(-2 \alpha^{2}+\alpha-3\right) f\left(\frac{3}{3 j}\right)}{2 j^{-\alpha}(3-\alpha)(2-\alpha)(1-\alpha)} \\
= & \left(\frac{1}{j}\right)^{-\alpha} \frac{1}{2(-\alpha)(-\alpha+1)(-\alpha+2)(-\alpha+3)}\left[\left(2 \alpha^{2}+8 \alpha+12\right) f(0)\right. \\
& \left.-\alpha(9 \alpha+27) f\left(\frac{1}{3 j}\right)+\left(18 \alpha^{2}\right) f\left(\frac{2}{3 j}\right)+\alpha\left(-2 \alpha^{2}+\alpha-3\right) f\left(\frac{3}{3 j}\right)\right] .
\end{aligned}
$$

Further, it is a simple matter to show that:

$$
\begin{aligned}
\int_{\frac{3 k-3}{3 j}}^{\frac{3 k}{3 j}} u^{-1-\alpha} P_{3}(u) d u= & \frac{1}{2 j^{-\alpha}(-\alpha)(-\alpha+1)(-\alpha+2)(-\alpha+3)}\left[F_{1}(k-1) f\left(\frac{3 k-3}{3 j}\right)\right. \\
& \left.+F_{2}(k) f\left(\frac{3 k-2}{3 j}\right)+F_{3}(k) f\left(\frac{3 k-1}{3 j}\right)+F_{0}(k) f\left(\frac{3 k}{3 j}\right)\right],
\end{aligned}
$$

in which $F_{i}(k), i=0,1,2,3$ are defined in (18). In this way the proof can easily be completed.

Remark 1. It is easy to show that

$$
w_{0,3 j}<0, \quad w_{1,3 j}>0, \quad w_{2,3 j}<0 .
$$


Also we have $w_{k, 3 j}>0$ for $k \geq 3$. To show the weights $w_{k, 3 j}>0, k=0,1, \ldots, 3 j$, we plot $w_{k, 15}>0$ in Fig. 1 for different values of $\alpha$.

Step 2 At the node $t_{l}=\frac{l}{3 m}$, with $l=3 j+1, j=1,2, \ldots, m-1$ :

$$
\mu y\left(t_{3 j+1}\right)=\frac{1}{\Gamma(-\alpha)} \oint_{0}^{t_{3 j+1}}\left(t_{3 j+1}-\tau\right)^{-1-\alpha} y(\tau) d \tau-\frac{y_{0}}{\Gamma(1-\alpha)} t_{3 j+1}^{-\alpha}-g\left(t_{3 j+1}\right),
$$

The integral in (5) can be written as

$$
\begin{gathered}
\oint_{0}^{t_{3 j+1}}\left(t_{3 j+1}-\tau\right)^{-1-\alpha} y(\tau) d \tau=\left[\oint_{0}^{t_{1}}+\oint_{t_{1}}^{t_{3 j+1}}\right]\left(t_{3 j+1}-\tau\right)^{-1-\alpha} y(\tau) d \tau \\
=\int_{0}^{t_{1}}\left(t_{3 j+1}-\tau\right)^{-1-\alpha} y(\tau) d \tau+t_{3 j+1}^{-\alpha} \oint_{0}^{\frac{3 j}{3 j+1}} u^{-1-\alpha} f(u) d u,
\end{gathered}
$$

here $f(u)=y\left(t_{3 j+1}-t_{3 j+1} u\right)$. The starting integral in Eq. 22 will be computed on the next subsection and the second integral is compute numerically in such a way:

$$
\oint_{0}^{\frac{3 j}{3 j+1}} u^{-1-\alpha} f(u) d u=\oint_{0}^{\frac{3 j}{3 j+1}} u^{-1-\alpha} P_{3}(u) d u+R_{3 j+1}(f) \approx I^{3 j+1},
$$

in which $R_{3 j+1}(f)$ is the reminder term and $P_{3}(u)$ is the piecewise cubic interpolation polynomial of $f(u)$ with the nodes $0, \frac{1}{3 j+1}, \frac{2}{3 j+1}, \ldots, \frac{3 j}{3 j+1}$. Set $f_{u}=y_{3 j+1-(3 j+1) \cdot u}$ be the numerical approximation of $f(u)$, then $I^{3 j+1}$ can be computed by repeating the calculations of Eqs. (8)-(9) under the hypotheses (10)-(14) with $l=3 j+1$.

Lemma 2. Fix $0<\alpha<1$. Then with $j=1,2, \ldots, m-1$, the integral in (23) yields

$$
\oint_{0}^{\frac{3 j}{3 j+1}} u^{-1-\alpha} P_{3}(u) d u=\sum_{k=0}^{3 j} w_{k, 3 j+1} f\left(\frac{k}{3 j+1}\right)=\sum_{k=0}^{3 j} w_{k, 3 j+1} y\left(t_{3 j+1-k}\right),
$$

${ }_{75}$ in which $w_{k, 3 j+1}=\left(\frac{3}{3 j+1}\right)^{-\alpha} \frac{1}{2(-\alpha)(-\alpha+1)(-\alpha+2)(-\alpha+3)} \tilde{w}_{k, 3 j}$ and $\tilde{w}_{k, 3 j}$ are given in Lemma 1

The proof is completely similar to the proof of Lemma 1

Step 3 At the node $t_{l}=\frac{l}{3 m}$, with $l=3 j+2, j=1,2, \ldots, m-1$ :

$$
\mu y\left(t_{3 j+2}\right)=\frac{1}{\Gamma(-\alpha)} \oint_{0}^{t_{3 j+2}}\left(t_{3 j+2}-\tau\right)^{-1-\alpha} y(\tau) d \tau-\frac{y_{0}}{\Gamma(1-\alpha)} t_{3 j+2}^{-\alpha}-g\left(t_{3 j+2}\right),
$$


The integral in (21) can be written as

$$
\begin{gathered}
\oint_{0}^{t_{3 j+2}}\left(t_{3 j+2}-\tau\right)^{-1-\alpha} y(\tau) d \tau=\left[\oint_{0}^{t_{2}}+\oint_{t_{2}}^{t_{3 j+2}}\right]\left(t_{3 j+2}-\tau\right)^{-1-\alpha} y(\tau) d \tau \\
=\int_{0}^{t_{2}}\left(t_{3 j+2}-\tau\right)^{-1-\alpha} y(\tau) d \tau+t_{3 j+2}^{-\alpha} \oint_{0}^{\frac{3 j}{3 j+2}} u^{-1-\alpha} f(u) d u,
\end{gathered}
$$

where $f(u)=y\left(t_{3 j+2}-t_{3 j+2} u\right)$. The starting integral in Eq. (26) will be computed on the next subsection and the second integral is compute numerically in such a way:

$$
\oint_{0}^{\frac{3 j}{3 j+2}} u^{-1-\alpha} f(u) d u=\oint_{0}^{\frac{3 j}{3 j+2}} u^{-1-\alpha} P_{3}(u) d u+R_{3 j+2}(f) \approx I^{3 j+2},
$$

in which $R_{3 j+2}(f)$ is the reminder term and $P_{3}(u)$ is the piecewise cubic interpolation polynomial of $f(u)$ with the nodes $0, \frac{1}{3 j+2}, \frac{2}{3 j+2}, \ldots, \frac{3 j}{3 j+2}$. Set $f_{u}=y_{3 j+2-(3 j+2) \cdot u}$ be

the numerical approximation of $f(u)$. Similar to the $I^{3 j}$ and $I^{3 j+1}$, the integral $I^{3 j+2}$ can be computed by repeating the calculations of Eqs. (8)-(9) under the hypotheses (10)-14) with $l=3 j+2$.

Lemma 3. Fix $0<\alpha<1$. Then with $j=1,2, \ldots, m-1$, the integral in (27) yields

$$
\oint_{0}^{\frac{3 j}{3 j+2}} u^{-1-\alpha} P_{3}(u) d u=\sum_{k=0}^{3 j} w_{k, 3 j+2} f\left(\frac{k}{3 j+2}\right)=\sum_{k=0}^{3 j} w_{k, 3 j+2} y\left(t_{3 j+2-k}\right),
$$

in which $w_{k, 3 j+2}=\left(\frac{3}{3 j+2}\right)^{-\alpha} \frac{1}{2(-\alpha)(-\alpha+1)(-\alpha+2)(-\alpha+3)} \tilde{w}_{k, 3 j}$ and $\tilde{w}_{k, 3 j}$ are given in Lemma 1

\subsection{Numerical estimation of the starting integrals}

This subsection deals with the approximation of the starting integrals in (22) and (26) with acceptable accuracy, which will be established in error analysis section3.

Remark 2. Since $j \geq 1$, the starting integrals $\int_{0}^{t_{1}}\left(t_{3 j+1}-\tau\right)^{-1-\alpha} y(\tau) d \tau$ and $\int_{0}^{t_{2}}\left(t_{3 j+2}-\right.$ $\tau)^{-1-\alpha} y(\tau) d \tau$ have no singular points on $\left[0, t_{1}\right]$ and $\left[0, t_{2}\right]$ respectively, so they are standard integrals.

For the starting integral in (22) we have

$$
I_{1}=\int_{0}^{t_{1}}\left(t_{3 j+1}-\tau\right)^{-1-\alpha} y(\tau) d \tau=t_{1} \int_{0}^{1}\left(t_{3 j}+t_{1} u\right)^{-1-\alpha} y\left(t_{1}-t_{1} u\right) d u
$$


Set $f\left(t_{1}\right)=\left(t_{3 j}+t_{1} u\right)$ and $q_{u}=y_{1-1 u}$ be the numerical approximation of $q(u)=y\left(t_{1}-\right.$ $\left.t_{1} u\right)$. The integral is replaced by the cubic interpolation polynomial of $q(u)$ on the nodes $0, \frac{1}{3}, \frac{2}{3}, 1$ then one can obtain

$$
\begin{aligned}
t_{1} \int_{0}^{1} f\left(t_{1}\right)^{-1-\alpha} q(u) d u & \approx t_{1} \int_{0}^{1} f\left(t_{1}\right)^{-1-\alpha}\left[l_{0}^{0}(u) q_{0}+l_{1}^{0}(u) q_{\frac{1}{3}}+l_{2}^{0}(u) q_{\frac{2}{3}}+l_{3}^{0}(u) q_{1}\right] d u \\
& =t_{1} \int_{0}^{1} f\left(t_{1}\right)^{-1-\alpha}\left[l_{0}^{0}(u) y_{1}+l_{1}^{0}(u) y_{\frac{2}{3}}+l_{2}^{0}(u) y_{\frac{1}{3}}+l_{3}^{0}(u) y_{0}\right] d u,
\end{aligned}
$$

where $l_{i}^{0}(u)$ are cubic interpolating functions, defined by

$$
\begin{cases}l_{0}^{0}(u)=\frac{\left(u-\frac{1}{3}\right)\left(u-\frac{2}{3}\right)(u-1)}{\left(0-\frac{1}{3}\right)\left(0-\frac{2}{3}\right)(0-1)}, & l_{1}^{0}(u)=\frac{(u-0)\left(u-\frac{2}{3}\right)(u-1)}{\left(\frac{1}{3}-0\right)\left(\frac{1}{3}-\frac{2}{3}\right)\left(\frac{1}{3}-1\right)} \\ l_{2}^{0}(u)=\frac{(u-0)\left(u-\frac{1}{3}\right)(u-1)}{\left(\frac{2}{3}-0\right)\left(\frac{2}{3}-\frac{1}{3}\right)\left(\frac{2}{3}-1\right)}, & l_{3}^{0}(u)=\frac{(u-0)\left(u-\frac{1}{3}\right)\left(u-\frac{2}{3}\right)}{(1-0)\left(1-\frac{1}{3}\right)\left(1-\frac{2}{3}\right)}\end{cases}
$$

The values of $y_{\frac{1}{3}}$ and $y_{\frac{2}{3}}$ are approximated by using the interpolation

$$
y_{\frac{1}{3}} \approx \frac{1}{81}\left(40 y_{0}+60 y_{1}-24 y_{2}+5 y_{3}\right), \quad y_{\frac{2}{3}} \approx \frac{1}{81}\left(14 y_{0}+84 y_{1}-21 y_{2}+4 y_{3}\right),
$$

Thus one can arrive the following numerical estimation for the starting integral in (22),

$$
\int_{0}^{t_{1}}\left(t_{3 j+1}-\tau\right)^{-1-\alpha} y(\tau) d \tau \approx t_{1} \sum_{k=0}^{3} W_{k, 3 j+1}^{0} y_{k}
$$

where

$$
\begin{cases}W_{0,3 j+1}^{0}=\frac{14}{81} \beta_{1,1}+\frac{40}{81} \beta_{2,1}+\beta_{3,1}, & W_{1,3 j+1}^{0}=\beta_{0,1}+\frac{28}{27} \beta_{1,1}+\frac{20}{27} \beta_{2,1} \\ W_{2,3 j+1}^{0}=-\frac{7}{27} \beta_{1,1}-\frac{8}{27} \beta_{2,1}, & W_{3,3 j+1}^{0}=\frac{4}{81} \beta_{1,1}+\frac{5}{81} \beta_{2,1}\end{cases}
$$

Denoting $f\left(t_{k}\right)=\left(t_{3 j}+t_{k} u\right)$ for $k=1,2$ we have

$$
\beta_{i, k}=\int_{0}^{1} f\left(t_{k}\right)^{-1-\alpha} l_{i}^{0}(u) d u=\rho h_{i}(k), \quad i=0,1,2,3 .
$$

This Integrals, after some calculations, leads to $\rho=\frac{3^{\alpha+3}\left(\frac{1}{m}\right)^{-\alpha-1}}{2 k^{4}(-\alpha)(-\alpha+1)(-\alpha+2)(-\alpha+3)}$ and

$$
\begin{aligned}
h_{0}(k)= & \frac{2}{9}(3 j+k)^{1-\alpha}\left[243 j^{2}+27 j k(\alpha+3)+k^{2}\left(\alpha^{2}+4 \alpha+6\right)\right]-3^{-\alpha-2} j^{-\alpha}\left[1458 j^{3}\right. \\
& \left.-324 j^{2} k(\alpha-3)+33 j k^{2}\left(\alpha^{2}-5 \alpha+6\right)-2 k^{3}\left(\alpha^{3}-6 \alpha^{2}+11 \alpha-6\right)\right]
\end{aligned}
$$




$$
\begin{gathered}
h_{1}(k)=-(3 j+k)^{1-\alpha}\left[162 j^{2}+12 j k(2 \alpha+3)+k^{2} \alpha(\alpha+3)\right] \\
+2 \times 3^{1-\alpha} j^{1-\alpha}\left[81 j^{2}-15 j k(\alpha-3)+k^{2}\left(\alpha^{2}-5 \alpha+6\right)\right] \\
h_{2}(k)=2(3 j+k)^{1-\alpha}\left[81 j^{2}+3 j k(5 \alpha+3)+\alpha^{2} k^{2}\right] \\
\quad-3^{1-\alpha} j^{1-\alpha}\left[162 j^{2}-24 j k(\alpha-3)+k^{2}\left(\alpha^{2}-5 \alpha+6\right)\right], \\
h_{3}(k)=-\frac{1}{9}(3 j+k)^{-\alpha}\left[1458 j^{3}+162 j^{2} k(2 \alpha+3)+3 j k^{2}\left(11 \alpha^{2}+17 \alpha+12\right)+\alpha k^{3}\right. \\
\left.\left(2 \alpha^{2}-\alpha+3\right)\right]+2 \times 3^{-\alpha-1} j^{1-\alpha}\left[243 j^{2}-27 j k(\alpha-3)+k^{2}\left(\alpha^{2}-5 \alpha+6\right)\right] .
\end{gathered}
$$

By comparing the Eqs. (33) and the value of the $I^{3 j+1}$ from step 2 one can construct the following numerical algorithm to approximate $y\left(t_{3 j+1}\right), j=1,2, \ldots, m-1$

$$
\begin{aligned}
\mu y_{3 j+1}= & \frac{t_{1}}{\Gamma(-\alpha)} \sum_{k=0}^{3} W_{k, 3 j+1}^{0} y_{k}+\frac{t_{3 j+1}^{-\alpha}}{\Gamma(-\alpha)} \sum_{k=1}^{j}\left[W_{3 k-3,3 j+1}^{k} y_{(3 j+1)-(3 k-3)}+W_{3 k-2,3 j+1}^{k} y_{(3 j+1)-(3 k-2)}\right. \\
& \left.+W_{3 k-1,3 j+1}^{k} y_{(3 j+1)-(3 k-1)}+W_{3 k, 3 j+1}^{k} y_{(3 j+1)-3 k}\right]-\frac{y_{0}}{\Gamma(1-\alpha)} t_{3 j+1}^{-\alpha}-g\left(t_{3 j+1}\right),
\end{aligned}
$$

The starting integral in (26) is calculate numerically in a quit similar way

$$
\begin{aligned}
& \int_{0}^{t_{2}}\left(t_{3 j+2}-\tau\right)^{-1-\alpha} y(\tau) d \tau=t_{2} \int_{0}^{1}\left(t_{3 j}+t_{2} u\right)^{-1-\alpha} q(u) d u \\
& \quad \approx t_{2} \int_{0}^{1} f\left(t_{2}\right)^{-1-\alpha}\left[l_{0}^{0}(u) q_{0}+l_{1}^{0}(u) q_{\frac{1}{3}}+l_{2}^{0}(u) q_{\frac{2}{3}}+l_{3}^{0}(u) q_{1}\right] d u \\
& \quad=t_{2} \int_{0}^{1} f\left(t_{2}\right)^{-1-\alpha}\left[l_{0}^{0}(u) y_{2}+l_{1}^{0}(u) y_{\frac{4}{3}}+l_{2}^{0}(u) y_{\frac{2}{3}}+l_{3}^{0}(u) y_{0}\right] d u,
\end{aligned}
$$

here $f\left(t_{2}\right)=\left(t_{3 j}+t_{2} u\right), q_{u}=y_{2-2 u}$ is the numerical approximation of $q(u)=y\left(t_{2}-t_{2} u\right)$, the value of $y_{\frac{2}{3}}$ is defined on (32) and $y_{\frac{4}{3}}$ is approximated by using the interpolation

$$
y_{\frac{4}{3}}=\frac{1}{81}\left(-5 y\left(t_{0}\right)+60 y\left(t_{1}\right)+30 y\left(t_{2}\right)-4 y\left(t_{3}\right)\right),
$$

Similar calculations performed above leads to the following numerical estimation,

$$
\int_{0}^{t_{2}}\left(t_{3 j+2}-\tau\right)^{-1-\alpha} y(\tau) d \tau \approx t_{2} \sum_{k=0}^{3} W_{k, 3 j+2}^{0} y_{k}
$$


where

$$
\begin{cases}W_{0,3 j+2}^{0}=-\frac{5}{81} \beta_{1,2}+\frac{14}{81} \beta_{2,2}+\beta_{3,2}, & W_{1,3 j+2}^{0}=\frac{20}{27} \beta_{1,2}+\frac{28}{27} \beta_{2,2}, \\ W_{2,3 j+2}^{0}=\beta_{0,2}+\frac{10}{27} \beta_{1,2}-\frac{7}{27} \beta_{2,2}, & W_{3,3 j+2}^{0}=-\frac{4}{81} \beta_{1,2}+\frac{4}{81} \beta_{2,2}\end{cases}
$$

with $\beta_{i, 2}=\rho h_{i}(2),(i=0,1,2,3)$ which is defined in (35). By comparing the Eqs. (43) and the value of the $I^{3 j+2}$ from step 3 one can design the following numerical algorithm to approximate $y\left(t_{3 j+2}\right), j=1,2, \ldots, m-1$

$$
\begin{aligned}
\mu y_{3 j+2}= & \frac{t_{2}}{\Gamma(-\alpha)} \sum_{k=0}^{3} W_{k, 3 j+2}^{0} y_{k}+\frac{t_{3 j+2}^{-\alpha}}{\Gamma(-\alpha)} \sum_{k=1}^{j}\left[W_{3 k-3,3 j+2}^{k} y_{(3 j+2)-(3 k-3)}+W_{3 k-2,3 j+2}^{k} y_{(3 j+2)-(3 k-2)}\right. \\
& \left.+W_{3 k-1,3 j+2}^{k} y_{(3 j+2)-(3 k-1)}+W_{3 k, 3 j+2}^{k} y_{(3 j+2)-3 k}\right]-\frac{y_{0}}{\Gamma(1-\alpha)} t_{3 j+2}^{-\alpha}-g\left(t_{3 j+2}\right),
\end{aligned}
$$

\subsection{Numerical estimation of the starting values}

At the node $t_{1}=\frac{1}{3 m}$, we have

$$
\mu y\left(t_{1}\right)=\frac{1}{\Gamma(-\alpha)} \oint_{0}^{t_{1}}\left(t_{1}-\tau\right)^{-1-\alpha} y(\tau) d \tau-\frac{y_{0}}{\Gamma(1-\alpha)} t_{1}^{-\alpha}-g\left(t_{1}\right)
$$

For the integral in Eq. (46) the following approximation is utilized:

$$
\begin{aligned}
I^{1} & =\oint_{0}^{t_{1}}\left(t_{1}-\tau\right)^{-1-\alpha} y(\tau) d \tau=t_{1}^{-\alpha} \oint_{0}^{1} u^{-1-\alpha} q(u) d u \\
& =t_{1}^{-\alpha} \oint_{0}^{1} u^{-1-\alpha}\left[l_{0}^{0} y\left(t_{1}\right)+l_{1}^{0} y\left(t_{\frac{2}{3}}\right)+l_{2}^{0} y\left(t_{\frac{1}{3}}\right)+l_{3}^{0} y\left(t_{0}\right)+R_{1}^{1}\right] d u,
\end{aligned}
$$

in which $q(u)$ is the same as $(30)$ and $R_{1}^{1}=\frac{g^{(4)}(c)}{4 !}(u-0)\left(u-\frac{1}{3}\right)\left(u-\frac{2}{3}\right)(u-1), c \in(0,1)$. Substituting the values of $y\left(t_{\frac{1}{3}}\right)$ and $y\left(t_{\frac{2}{3}}\right)$ from (32) leads to

$$
I^{1}=t_{1}^{-\alpha}\left[\sum_{k=0}^{3} w_{k, 1} y\left(t_{k}\right)+\oint_{0}^{1} u^{-1-\alpha}\left[R_{1}^{1}+R^{\frac{1}{3}}+R^{\frac{2}{3}}\right] d u\right]=t_{1}^{-\alpha} \sum_{k=0}^{3} w_{k, 1} y\left(t_{k}\right)+R_{1}^{3},
$$

where $R^{\frac{1}{3}}=-\frac{10 y^{(4)}(c)}{243} h^{4}, R^{\frac{2}{3}}=-\frac{7 y^{(4)}(c)}{243} h^{4}$ and $R_{1}^{3}$ will be calculated on the next section.

$$
\begin{cases}w_{0,1}=\frac{14}{81} \beta_{1}+\frac{40}{81} \beta_{2}+\beta_{3}, & w_{1,1}=\beta_{0}+\frac{28}{27} \beta_{1}+\frac{20}{27} \beta_{2} \\ w_{2,1}=-\frac{7}{27} \beta_{1}-\frac{8}{27} \beta_{2}, & w_{3,1}=\frac{4}{81} \beta_{1}+\frac{5}{81} \beta_{2}\end{cases}
$$


with

$$
\beta_{i}=\oint_{0}^{1} u^{-1-\alpha} l_{i}^{0}(u) d u, \quad i=0,1,2,3 .
$$

The cubic Lagrange polynomials $l_{i}^{0}(u)$ are defined in 31 . So the following numerical estimation is straightforward

$$
\mu y_{1}=\frac{t_{1}^{-\alpha}}{\Gamma(-\alpha)} \sum_{k=0}^{3} w_{k, 1} y_{k}-\frac{y_{0}}{\Gamma(1-\alpha)} t_{1}^{-\alpha}-g\left(t_{1}\right),
$$

Lemma 4. Fix $0<\alpha<1$. Then the weights $\beta_{i}$ in (50) are satisfied:

$$
\beta_{i}=\frac{1}{2(-\alpha)(-\alpha+1)(-\alpha+2)(-\alpha+3)} \begin{cases}2\left(\alpha^{2}+4 \alpha+6\right), & \text { for } i=0, \\ -9 \alpha(\alpha+3), & \text { for } i=1, \\ 18 \alpha^{2}, & \text { for } i=2, \\ \alpha\left(-2 \alpha^{2}+\alpha-3\right), & \text { for } i=3,\end{cases}
$$

Proof. We will just calculate $\beta_{0}$, the other cases can be achieved in the same manner. Definition of the Hadamard finite-part integral leads to

$$
\begin{gathered}
\beta_{0}=\oint_{0}^{1} u^{-1-\alpha} l_{0}^{0}(u) d u=\frac{l_{0}^{0}(0) 1^{-\alpha}}{-\alpha}+\int_{0}^{1} u^{-1-\alpha}(I I) d u \\
I I=\int_{0}^{u}\left(l_{0}^{0}\right)^{\prime}(y) d y=\int_{0}^{u} \frac{1}{2}\left(-27 y^{2}+36 y-11\right) d y=-\frac{9 u^{3}}{2}+9 u^{2}-\frac{11 u}{2},
\end{gathered}
$$

substituting (54) into (53) and calculating the integral yields

$$
\beta_{0}=\frac{1}{-\alpha}+\frac{\alpha^{2}-5 \alpha+15}{(\alpha-3)(\alpha-2)(\alpha-1)}=\frac{\alpha^{2}+4 \alpha+6}{(-\alpha)(-\alpha+1)(-\alpha+2)(-\alpha+3)} .
$$

At the node $t_{2}=\frac{2}{3 m}$, we have

$$
\mu y\left(t_{2}\right)=\frac{1}{\Gamma(-\alpha)} \oint_{0}^{t_{2}}\left(t_{2}-\tau\right)^{-1-\alpha} y(\tau) d \tau-\frac{y_{0}}{\Gamma(1-\alpha)} t_{2}^{-\alpha}-g\left(t_{2}\right) .
$$

The following approximation is applied for the integral in Eq.(55)

$$
\begin{aligned}
I^{2} & =\oint_{0}^{t_{2}}\left(t_{2}-\tau\right)^{-1-\alpha} y(\tau) d \tau=t_{2}^{-\alpha} \oint_{0}^{1} u^{-1-\alpha} q(u) d u \\
& =t_{2}^{-\alpha} \oint_{0}^{1} f\left(t_{2}\right)^{-1-\alpha}\left[l_{0}^{0} y\left(t_{1}\right)+l_{1}^{0} y\left(t_{4}\right)+l_{2}^{0} y\left(t_{2}\right)+l_{3}^{0} y\left(t_{0}\right)+R_{1}^{1}\right] d u,
\end{aligned}
$$


here $q(u)$ and $R_{1}^{1}$ are the same as (41) and (47) respectively. Substituting the values of $y\left(t_{\frac{2}{3}}\right)$ and $y\left(t_{\frac{4}{3}}\right)$ from (32) and (42) leads to

$$
I^{2}=t_{2}^{-\alpha}\left[\sum_{k=0}^{3} w_{k, 2} y\left(t_{k}\right)+\oint_{0}^{1} u^{-1-\alpha}\left[R_{1}^{1}+R^{\frac{2}{3}}+R^{\frac{4}{3}}\right] d u\right]=t_{2}^{-\alpha} \sum_{k=0}^{3} w_{k, 2} y\left(t_{k}\right)+R_{2}^{1},
$$

where $R^{\frac{4}{3}}=\frac{5 y^{(4)}(c)}{243} h^{4}$ and $R_{2}^{1}$ will be calculated on the next section.

$$
\begin{cases}w_{0,2}=-\frac{5}{81} \beta_{1}+\frac{14}{81} \beta_{2}+\beta_{3}, & w_{1,2}=\frac{20}{27} \beta_{1}+\frac{28}{27} \beta_{2}, \\ w_{2,2}=\beta_{0}+\frac{10}{27} \beta_{1}-\frac{7}{27} \beta_{2}, & w_{3,2}=-\frac{4}{81} \beta_{1}+\frac{4}{81} \beta_{2},\end{cases}
$$

in which $\beta_{i}, i=0,1,2,3$ are given in the Lemma4,

Hence, we arrive the following numerical estimation

$$
\mu y_{2}=\frac{t_{2}^{-\alpha}}{\Gamma(-\alpha)} \sum_{k=0}^{3} w_{k, 2} y_{k}-\frac{y_{0}}{\Gamma(1-\alpha)} t_{2}^{-\alpha}-g\left(t_{2}\right)
$$

\subsection{The novel high-order algorithm}

In this subsection, the results of the previous subsections are used to design the following overall novel algorithm for the numerical estimation of (2).

$$
\begin{cases}y_{l}=\frac{1}{w_{l, l}-t_{l}^{\alpha} \Gamma(-\alpha) \mu}\left[t_{l}^{\alpha} \Gamma(-\alpha) g\left(t_{l}\right)-\sum_{\substack{k=0 \\ k \neq i}}^{3} \tilde{\tilde{w}}_{k, l} y_{k}\right], & \text { for } l=1,2, \\ y_{l}=\frac{1}{w_{0, l}-t_{l}^{\alpha} \Gamma(-\alpha) \mu}\left[t_{l}^{\alpha} \Gamma(-\alpha) g\left(t_{l}\right)-\sum_{k=1}^{l} \tilde{\tilde{w}}_{k, l} y_{l-k}\right], & \text { for } l=3 j, 3 j+1,3 j+2,\end{cases}
$$

where

$$
\begin{gathered}
\text { with } l=1,2: \tilde{\tilde{w}}_{k, l}= \begin{cases}\frac{1}{\alpha}+w_{0, l}, & k=0, \\
w_{k, l}, & k=1,2,3,\end{cases} \\
\text { with } l=3 j, j=1, \ldots, m: \tilde{w}_{k, 3 j}= \begin{cases}w_{k, 3 j}, & k=1, \ldots, 3 j-1, \\
\frac{1}{\alpha}+w_{3 j, 3 j}, & k=3 j,\end{cases}
\end{gathered}
$$


with $l=3 j+1, j=1, \ldots, m-1: \tilde{\tilde{w}}_{k, 3 j+1}= \begin{cases}w_{k, 3 j+1}, & k=1, \ldots, 3 j-3, \\ w_{3 j-2,3 j+1}+t_{3 j+1}^{\alpha} t_{1} W_{3,3 j+1}^{0}, & k=3 j-2, \\ w_{3 j-1,3 j+1}+t_{3 j+1}^{\alpha} t_{1} W_{2,3 j+1}^{0}, & k=3 j-1, \\ w_{3 j, 3 j+1}+t_{3 j+1}^{\alpha} t_{1} W_{1,3 j+1}^{0}, & k=3 j, \\ \frac{1}{\alpha}+t_{3 j+1}^{\alpha} t_{1} W_{0,3 j+1}^{0}, & k=3 j+1,\end{cases}$
with $l=3 j+2, j=1, \ldots, m-1: \tilde{\tilde{w}}_{k, 3 j+2}= \begin{cases}w_{k, 3 j+2}, & k=1, \ldots, 3 j-2, \\ w_{3 j-1,3 j+2}+t_{3 j+2}^{\alpha} t_{2} W_{3,3 j+2}^{0}, & k=3 j-1, \\ w_{3 j, 3 j+2}+t_{3 j+2}^{\alpha} t_{2} W_{2,3 j+2}^{0}, & k=3 j, \\ t_{3 j+2}^{\alpha} t_{2} W_{1,3 j+2}^{0}, & k=3 j+1, \\ \frac{1}{\alpha}+t_{3 j+2}^{\alpha} t_{2} W_{0,3 j+2}^{0}, & k=3 j+2,\end{cases}$

95 It is obvious that, the first three step solutions $y_{1}, y_{2}$ and $y_{3}$ are coupled in (59), thus need to be solved simultaneously. An explicit solution of these equations is given in Appendix section.

\section{Truncation error analysis}

For the presented numerical algorithm the truncation error at the node $t_{i}$ is defined by [37]

$$
E_{l}(h):=y\left(t_{l}\right)-\tilde{y}_{l}, \quad l=3 j, 3 j+1,3 j+2, j \geq 1 .
$$

where $\tilde{y}_{l}$ is an approximation to $y\left(t_{l}\right)$, evaluated by using the presented numerical algorithm with exact previous solutions, i.e. for $j \geq 1$,

$$
\begin{aligned}
\mu \tilde{y}_{3 j}= & \frac{t_{3 j}^{-\alpha}}{\Gamma(-\alpha)} \sum_{k=1}^{j}\left[W_{3 k-3,3 j}^{k} y\left(t_{3 j-(3 k-3)}\right)+W_{3 k-2,3 j}^{k} y\left(t_{3 j-(3 k-2)}\right)+W_{3 k-1,3 j}^{k} y\left(t_{3 j-(3 k-1)}\right)\right. \\
& \left.+W_{3 k, 3 j}^{k} y\left(t_{3 j-3 k}\right)\right]-\frac{y_{0}}{\Gamma(1-\alpha)} t_{3 j}^{-\alpha}-g\left(t_{3 j}\right)
\end{aligned}
$$




$$
\begin{aligned}
\mu \tilde{y}_{3 j+1}= & \frac{t_{1}}{\Gamma(-\alpha)} \sum_{k=0}^{3} W_{k, 3 j+1}^{0} y\left(t_{k}\right)+\frac{t_{3 j+1}^{-\alpha}}{\Gamma(-\alpha)} \sum_{k=1}^{j}\left[W_{3 k-3,3 j+1}^{k} y\left(t_{(3 j+1)-(3 k-3)}\right)\right. \\
& +W_{3 k-2,3 j+1}^{k} y\left(t_{(3 j+1)-(3 k-2)}\right)+W_{3 k-1,3 j+1}^{k} y\left(t_{(3 j+1)-(3 k-1)}\right) \\
& \left.+W_{3 k, 3 j+1}^{k} y\left(t_{(3 j+1)-3 k}\right)\right]-\frac{y_{0}}{\Gamma(1-\alpha)} t_{3 j+1}^{-\alpha}-g\left(t_{3 j+1}\right), \\
\mu \tilde{y}_{3 j+2}= & \frac{t_{2}}{\Gamma(-\alpha)} \sum_{k=0}^{3} W_{k, 3 j+2}^{0} y\left(t_{k}\right)+\frac{t_{3 j+2}^{-\alpha}}{\Gamma(-\alpha)} \sum_{k=1}^{j}\left[W_{3 k-3,3 j+2}^{k} y\left(t_{(3 j+2)-(3 k-3)}\right)\right. \\
& +W_{3 k-2,3 j+2}^{k} y\left(t_{(3 j+2)-(3 k-2)}\right)+W_{3 k-1,3 j+2}^{k} y\left(t_{(3 j+2)-(3 k-1)}\right) \\
& \left.+W_{3 k, 3 j+2}^{k} y\left(t_{(3 j+2)-3 k}\right)\right]-\frac{y_{0}}{\Gamma(1-\alpha)} t_{3 j+2}^{-\alpha}-g\left(t_{3 j+2}\right),
\end{aligned}
$$

Throughout this subsection it is assumed that $y(t) \in C^{4}[0,1]$.

Lemma 5. There exist constants $C_{1}$ and $C_{2}$ such that the reminder terms $R_{1}^{3}$ in (48) and $R_{2}^{1}$ in (56) are satisfied $\left|R_{1}^{3}\right| \leq C_{1} h^{4-\alpha}$ and $\left|R_{2}^{1}\right| \leq C_{2} h^{4-\alpha}$.

Proof. Set $q(u)=y\left(t_{1}-t_{1} u\right)$.

$$
\begin{aligned}
\left|R_{1}^{3}\right| & \leq\left|t_{1}^{-\alpha} \int_{0}^{1} u^{-1-\alpha} R_{1}^{1} d u\right|+\left|t_{1}^{-\alpha} \int_{0}^{1} u^{-1-\alpha}\left(R^{\frac{1}{3}}+R^{\frac{2}{3}}\right) d u\right| \\
& \leq t_{1}^{-\alpha} \frac{\left\|q^{(4)}\right\|_{\infty}}{4 !}\left(\tilde{u}-\frac{1}{3}\right)\left(\tilde{u}-\frac{2}{3}\right)(\tilde{u}-1) \int_{0}^{1} u^{-\alpha} d u+t_{1}^{-\alpha}\left|C_{3}\left\|y^{(4)}\right\|_{\infty} h^{4} \oint_{0}^{1} u^{-1-\alpha} d u\right|,
\end{aligned}
$$

here $\tilde{u} \in[0,1]$ and the second integral mean value theorem is used. Recall that $\oint_{0}^{1} u^{-1-\alpha} d u=$ $\frac{-1}{\alpha}$ [28].

$$
\left|R_{1}^{3}\right| \leq t_{1}^{-\alpha}\left(C_{4}\left\|q^{(4)}\right\|_{\infty} \frac{1}{-\alpha+1}+C_{3}\left\|y^{(4)}\right\|_{\infty} h^{4}\left|\frac{-1}{\alpha}\right|\right)
$$

Let $w=\left(t_{1}-t_{1} u\right)$, then

$$
\frac{d q(u)}{d u}=\frac{d y(w)}{d w} \frac{d w}{d u}=-t_{1} \frac{d y(w)}{d w} \Rightarrow \frac{d^{4} q(u)}{d u^{4}}=t_{1}^{4} \frac{d^{4} y(w)}{d w^{4}},
$$

utilizing this, it can be checked out that

$$
\left|R_{1}^{3}\right| \leq t_{1}^{-\alpha}\left(C_{4} t_{1}^{4}\left\|y^{(4)}\right\|_{\infty} \frac{1}{-\alpha+1}+C_{3}\left\|y^{(4)}\right\|_{\infty} h^{4}\left|\frac{-1}{\alpha}\right|\right) \leq C_{1} h^{4-\alpha}
$$

Using similar analysis it can be shown that $\left|R_{2}^{1}\right| \leq C_{2} h^{4-\alpha}$. 
Theorem 1. Set $E_{l}(h), l=3 j$ being the truncation error at the node $t_{i}$ defined in (60), then it holds

$$
\left|E_{l}(h)\right| \leq C h^{4-\alpha}, \quad l=3 j, j=1,2,3, \ldots, m
$$

Proof. Set $f(u)=y\left(t_{l}-t_{l} u\right)$, by comparing Eqs. (5)-(9) with $l=3 j$ and (61), it follows that

$$
\begin{aligned}
& \mu y\left(t_{l}\right)-\mu \tilde{y}_{l}= \frac{t_{l}^{-\alpha}}{\Gamma(-\alpha)} \oint_{0}^{1} u^{-1-\alpha} f(u) d u-\frac{t_{l}^{-\alpha}}{\Gamma(-\alpha)} \sum_{k=1}^{j}\left[W_{3 k-3, l}^{k} y\left(t_{l-(3 k-3)}\right)\right. \\
&\left.+W_{3 k-3,2}^{k} y\left(t_{l-(3 k-2)}\right)+W_{3 k-1, l}^{k} y\left(t_{l-(3 k-1)}\right)+W_{3 k, l}^{k} y\left(t_{l-3 k}\right)\right] \\
&= \frac{t_{l}^{-\alpha}}{\Gamma(-\alpha)}\left[\left(\oint_{0}^{\frac{3}{T}}+\sum_{k=2}^{j} \int_{\frac{3 k-3}{l}}^{\frac{3 k}{l}}\right) u^{-1-\alpha} f(u) d u-\sum_{k=1}^{j}\left[W_{3 k-3, l}^{k} f\left(\frac{3 k-3}{l}\right)\right.\right. \\
&\left.\left.+W_{3 k-2, l}^{k} f\left(\frac{3 k-2}{l}\right)+W_{3 k-1, l}^{k} f\left(\frac{3 k-1}{l}\right)+W_{3 k, l}^{k} f\left(\frac{3 k}{l}\right)\right]\right] \\
& \mu\left(y\left(t_{l}\right)-\tilde{y}_{l}\right)=\frac{t_{l}^{-\alpha}}{\Gamma(-\alpha)}\left[\oint_{0}^{\frac{3}{T}} u^{-1-\alpha} R_{1}(u) d u+\sum_{k=2}^{j} \int_{\frac{3 k-3}{l}}^{\frac{3 k}{T}} u^{-1-\alpha} R_{k}(u) d u,\right.
\end{aligned}
$$

in which

$$
\begin{aligned}
R_{k}(u)= & f(u)-\left[l_{3 k-3, l}^{k}(u) f\left(\frac{3 k-3}{l}\right)+l_{3 k-2, l}^{k}(u) f\left(\frac{3 k-2}{l}\right)+l_{3 k-1, l}^{k}(u) f\left(\frac{3 k-1}{l}\right)\right. \\
& \left.+l_{3 k, l}^{k}(u) f\left(\frac{3 k}{l}\right)\right], \quad k=1,2, \ldots, j .
\end{aligned}
$$

By using Taylor theorem for all $u \in\left[\frac{3 k-3}{l}, \frac{3 k}{l}\right]$ there exist $c_{k} \in\left[\frac{3 k-3}{l}, \frac{3 k}{l}\right]$, such that

$$
R_{k}(u)=\frac{f^{(4)}\left(c_{k}\right)}{4 !}\left(u-\frac{3 k-3}{l}\right)\left(u-\frac{3 k-2}{l}\right)\left(u-\frac{3 k-1}{l}\right)\left(u-\frac{3 k}{l}\right),
$$

In this way, one can obtain

$$
\mu\left(y\left(t_{l}\right)-\tilde{y}_{l}\right)=\frac{t_{l}^{-\alpha}}{\Gamma(-\alpha)}\left(I_{1}+I_{2}\right),
$$

where for $\tilde{u}_{1} \in\left[0, \frac{3}{l}\right]$, we have

$$
\begin{aligned}
\left|I_{1}\right| & =\left|\oint_{0}^{\frac{3}{l}} u^{-1-\alpha} \frac{f^{(4)}\left(c_{k}\right)}{4 !}(u-0)\left(u-\frac{1}{l}\right)\left(u-\frac{2}{l}\right)\left(u-\frac{3}{l}\right) d u\right| \\
& \leq \frac{\left\|f^{(4)}\right\|_{\infty}}{4 !}\left(\tilde{u}_{1}-\frac{1}{l}\right)\left(\tilde{u}_{1}-\frac{2}{l}\right)\left(\tilde{u}_{1}-\frac{3}{l}\right) \int_{0}^{\frac{3}{T}} u^{-\alpha} d u \leq C_{1}\left(\frac{1}{l}\right)^{3}\left\|f^{(4)}\right\|_{\infty}\left(\frac{3}{l}\right)^{1-\alpha} .
\end{aligned}
$$


For $\tilde{u}_{k} \in\left[\frac{3 k-3}{l}, \frac{3 k}{l}\right]$, it can be checked out that

$$
\begin{gathered}
\left|I_{2}\right|=\left|\sum_{k=2}^{j} \int_{\frac{3 k-3}{l}}^{\frac{3 k}{l}} u^{-1-\alpha} \frac{f^{(4)}\left(c_{k}\right)}{4 !}\left(u-\frac{3 k-3}{l}\right)\left(u-\frac{3 k-2}{l}\right)\left(u-\frac{3 k-1}{l}\right)\left(u-\frac{3 k}{l}\right) d u\right| \\
\leq \sum_{k=2}^{j} \frac{f^{(4)}\left(c_{k}\right)}{4 !}\left(\tilde{u}_{k}-\frac{3 k-3}{l}\right)\left(\tilde{u}_{k}-\frac{3 k-2}{l}\right)\left(\tilde{u}_{k}-\frac{3 k-1}{l}\right)\left(\tilde{u}_{k}-\frac{3 k}{l}\right) \int_{\frac{3 k-3}{l}}^{\frac{3 k}{l}} u^{-1-\alpha} d u, \\
\left|I_{2}\right| \leq C_{3}\left(\frac{1}{l}\right)^{4}\left\|f^{(4)}\right\|_{\infty} \sum_{k=2}^{j} \frac{1}{\alpha}\left[\left(\frac{3 k-3}{l}\right)^{-\alpha}-\left(\frac{3 k}{l}\right)^{-\alpha}\right] \\
=C_{3}\left(\frac{1}{l}\right)^{4}\left\|f^{(4)}\right\|_{\infty}\left[\left(\frac{3}{l}\right)^{-\alpha}-\left(\frac{3 j}{l}\right)^{-\alpha}\right] \leq C_{3}\left(\frac{1}{l}\right)^{4}\left\|f^{(4)}\right\|_{\infty}\left(\frac{3}{l}\right)^{-\alpha} .
\end{gathered}
$$

Together these estimates, for $l=3 j$ one can conclude that

$$
\begin{aligned}
\left|E_{l}(h)\right| & =\frac{t_{l}^{-\alpha}}{|\mu \Gamma(-\alpha)|}\left(\left|I_{1}\right|+\left|I_{2}\right|\right) \leq \frac{\left(\frac{l}{3 m}\right)^{-\alpha}}{|\mu \Gamma(-\alpha)|}\left[C_{5}\left(\frac{1}{l}\right)^{4-\alpha}\left\|f^{(4)}\right\|_{\infty}\right] \\
& \leq C_{6}\left(\frac{1}{l}\right)^{4}\left(\frac{1}{3 m}\right)^{-\alpha}\left\|f^{(4)}\right\|_{\infty},
\end{aligned}
$$

Similarly to 655) for $w=\left(t_{l}-t_{l} u\right)$ it can be shown that $\frac{d^{4} f(u)}{d u^{4}}=t_{l}^{4} \frac{d^{4} y(w)}{d w^{4}}$. For this reason one can obtain

$$
\left|E_{i}(h)\right| \leq C_{6}\left(\frac{1}{l}\right)^{4}\left(\frac{1}{3 m}\right)^{-\alpha}\left(\frac{l}{3 m}\right)^{4}\left\|y^{(4)}\right\|_{\infty} \leq C\left(\frac{1}{3 m}\right)^{4-\alpha}=C h^{4-\alpha} .
$$

Theorem 2. Set $E_{l}(h), l=3 j+1$ being the truncation error at the node $t_{l}$ defined in (60), then it holds

$$
\left|E_{l}(h)\right| \leq C h^{4-\alpha}, \quad l=3 j+1, j=1,2,3, \ldots, m-1
$$

Proof. By comparing Eqs. (21), (22) and (62) one can obtain

$$
\begin{aligned}
\mu y\left(t_{l}\right)-\mu \tilde{y}_{l}= & \frac{1}{\Gamma(-\alpha)} \int_{0}^{t_{1}}\left(t_{3 j+1}-\tau\right)^{-1-\alpha} y(\tau) d \tau+\frac{t_{3 j+1}^{-\alpha}}{\Gamma(-\alpha)} \oint_{0}^{\frac{3 j}{3 j+1}} u^{-1-\alpha} f(u) d u \\
& -\frac{t_{1}}{\Gamma(-\alpha)} \sum_{k=0}^{3} W_{k, 3 j+1}^{0} y\left(t_{k}\right)-\frac{t_{3 j+1}^{-\alpha}}{\Gamma(-\alpha)} \sum_{k=1}^{j}\left[W_{3 k-3,3 j+1}^{k} y\left(t_{(3 j+1)-(3 k-3)}\right)\right. \\
& +W_{3 k-2,3 j+1}^{k} y\left(t_{(3 j+1)-(3 k-2)}\right)+W_{3 k-1,3 j+1}^{k} y\left(t_{(3 j+1)-(3 k-1)}\right) \\
& \left.+W_{3 k, 3 j+1}^{k} y\left(t_{(3 j+1)-3 k}\right)\right]
\end{aligned}
$$


combining (29), (34) and (8)-(9) with $l=3 j+1$, and, we have

$$
\begin{aligned}
\mu E_{l}(h)= & \frac{t_{1}}{\Gamma(-\alpha)} \int_{0}^{1} f\left(t_{1}\right)^{-1-\alpha} q(u) d u+\frac{t_{3 j+1}^{-\alpha}}{\Gamma(-\alpha)}\left[\oint_{0}^{\frac{3}{T}}+\sum_{k=2}^{j} \int_{\frac{3 k-3}{l}}^{\frac{3 k}{T}}\right] u^{-1-\alpha} f(u) d u \\
& -\frac{t_{1}}{\Gamma(-\alpha)}\left[\left(\frac{14}{81} \beta_{1,1}+\frac{40}{81} \beta_{2,1}+\beta_{3,1}\right) y\left(t_{0}\right)-\left(\beta_{0,1}+\frac{28}{27} \beta_{1,1}+\frac{20}{27} \beta_{2,1}\right) y\left(t_{1}\right)\right. \\
& \left.-\left(-\frac{7}{27} \beta_{1,1}-\frac{8}{27} \beta_{2,1}\right) y\left(t_{2}\right)-\left(\frac{4}{81} \beta_{1,1}+\frac{5}{81} \beta_{2,1}\right) y\left(t_{3}\right)\right]-\frac{t_{3 j+1}^{-\alpha}}{\Gamma(-\alpha)} \\
& \sum_{k=1}^{j}\left[W_{3 k-3, l}^{k} f\left(\frac{3 k-3}{l}\right)+W_{3 k-2, l}^{k} f\left(\frac{3 k-2}{l}\right)+W_{3 k-1, l}^{k} f\left(\frac{3 k-1}{l}\right)\right. \\
& \left.+W_{3 k, l}^{k} f\left(\frac{3 k}{l}\right)\right]=I_{1}+I I_{1},
\end{aligned}
$$

with

$$
\begin{aligned}
\frac{\Gamma(-\alpha)}{t_{1}} I_{1}= & \int_{0}^{1} f\left(t_{1}\right)^{-1-\alpha} q(u) d u-\left[\beta_{0,1} y\left(t_{1}\right)+\beta_{1,1} y\left(t_{\frac{2}{3}}\right)+\beta_{2,1} y\left(t_{\frac{1}{3}}\right)+\beta_{3,1} y\left(t_{0}\right)\right] \\
& +\beta_{1,1}\left[y\left(t_{\frac{2}{3}}\right)-\frac{1}{81}\left(14 y\left(t_{0}\right)+84 y\left(t_{1}\right)-21 y\left(t_{2}\right)+4 y\left(t_{3}\right)\right)\right] \\
& +\beta_{2,1}\left[y\left(t_{\frac{1}{3}}\right)-\frac{1}{81}\left(40 y\left(t_{0}\right)+60 y\left(t_{1}\right)-24 y\left(t_{2}\right)+5 y\left(t_{3}\right)\right)\right], \\
\frac{\Gamma(-\alpha)}{t_{l}^{-\alpha}} I I_{1}= & \oint_{0}^{\frac{3}{T}} u^{-1-\alpha}\left(f(u)-\left[l_{0, l}^{1}(u) f(0)+l_{1, l}^{1}(u) f\left(\frac{1}{l}\right)+l_{2, l}^{1}(u) f\left(\frac{2}{l}\right)\right.\right. \\
& \left.\left.+l_{3, l}^{1}(u) f\left(\frac{3}{l}\right)\right]\right) d u+\sum_{k=2}^{j} \int_{\frac{3 k-3}{l}}^{\frac{3 k}{T}} u^{-1-\alpha}\left(f(u)-\left[l_{3 k-3, l}^{k}(u) f\left(\frac{3 k-3}{l}\right)\right.\right. \\
& \left.\left.+l_{3 k-2, l}^{k}(u) f\left(\frac{3 k-2}{l}\right)+l_{3 k-1, l}^{k}(u) f\left(\frac{3 k-1}{l}\right)+l_{3 k, l}^{k}(u) f\left(\frac{3 k}{l}\right)\right]\right) d u,
\end{aligned}
$$

Analysis similar to that in the proof of Theorem 1 shows that $\left|I I_{1}\right| \leq C_{4} h^{4-\alpha}$. For $I_{1}$ from (35) it can be checked out that

$$
\frac{\Gamma(-\alpha)}{t_{1}} I_{1}=\int_{0}^{1} f\left(t_{1}\right)^{-1-\alpha}\left(R^{1}(u)+R^{\frac{2}{3}}(u)+R^{\frac{1}{3}}(u)\right) d u
$$


where

$$
\begin{aligned}
& R^{1}(u)= p(u)-\left[l_{0}^{0} y\left(t_{1}\right)+l_{1}^{0} y\left(t_{\frac{2}{3}}\right)+l_{2}^{0} y\left(t_{\frac{1}{3}}\right)+l_{3}^{0} y\left(t_{0}\right)\right]=q(u)-\left[l_{0}^{0} q(1)+l_{1}^{0} q\left(\frac{1}{3}\right)\right. \\
&\left.+l_{2}^{0} q\left(\frac{2}{3}\right)+l_{3}^{0} q(0)\right]=\frac{q^{(4)}(c)}{4 !}(u-0)\left(u-\frac{1}{3}\right)\left(u-\frac{2}{3}\right)(u-1), \quad u, c \in[0,1], \\
& R^{\frac{2}{3}}(u)=l_{1}^{0}\left[y\left(t_{\frac{2}{3}}\right)-\frac{1}{81}\left(14 y\left(t_{0}\right)+84 y\left(t_{1}\right)-21 y\left(t_{2}\right)+4 y\left(t_{3}\right)\right)\right]=l_{1}^{0}\left[-\frac{7 y^{(4)}(c)}{243} h^{4}\right], \\
& R^{\frac{1}{3}}(u)=l_{2}^{0}\left[y\left(t_{\frac{1}{3}}\right)-\frac{1}{81}\left(40 y\left(t_{0}\right)+60 y\left(t_{1}\right)-24 y\left(t_{2}\right)+5 y\left(t_{3}\right)\right)\right]=l_{2}^{0}\left[-\frac{10 y^{(4)}(c)}{243} h^{4}\right] .
\end{aligned}
$$

In this way, for it is found that

$$
\begin{aligned}
& I_{1}= \frac{t_{1}}{\Gamma(-\alpha)} \int_{0}^{1} f\left(t_{1}\right)^{-1-\alpha}\left[\frac{q^{(4)}(c)}{4 !}(u-0)\left(u-\frac{1}{3}\right)\left(u-\frac{2}{3}\right)(u-1)\right] d u \\
&+\frac{t_{1}}{\Gamma(-\alpha)} \int_{0}^{1} f\left(t_{1}\right)^{-1-\alpha}\left[-\frac{7 y^{(4)}(c)}{243} h^{4}\right] \frac{(u-0)\left(u-\frac{2}{3}\right)(u-1)}{\left(\frac{1}{3}-0\right)\left(\frac{1}{3}-\frac{2}{3}\right)\left(\frac{1}{3}-1\right)} d u \\
&+\frac{t_{1}}{\Gamma(-\alpha)} \int_{0}^{1} f\left(t_{1}\right)^{-1-\alpha}\left[-\frac{10 y^{(4)}(c)}{243} h^{4}\right] \frac{(u-0)\left(u-\frac{1}{3}\right)(u-1)}{\left(\frac{2}{3}-0\right)\left(\frac{2}{3}-\frac{1}{3}\right)\left(\frac{2}{3}-1\right)} d u \\
&\left|I_{1}\right| \leq \frac{t_{1}}{\Gamma(-\alpha)} \frac{\left\|q^{(4)}\right\|_{\infty}}{4 !}(\tilde{u}-0)\left(\tilde{u}-\frac{1}{3}\right)\left(\tilde{u}-\frac{2}{3}\right)(\tilde{u}-1) \int_{0}^{1} f\left(t_{1}\right)^{-1-\alpha} d u \\
&+\frac{t_{1}}{\Gamma(-\alpha)} \frac{7 y^{(4)}(c)}{243} h^{4} \frac{(\tilde{u}-0)\left(\tilde{u}-\frac{2}{3}\right)(\tilde{u}-1)}{\left(\frac{1}{3}-0\right)\left(\frac{1}{3}-\frac{2}{3}\right)\left(\frac{1}{3}-1\right)} \int_{0}^{1} f\left(t_{1}\right)^{-1-\alpha} d u \\
&+\frac{t_{1}}{\Gamma(-\alpha)} \frac{10 y^{(4)}(c)}{243} h^{4} \frac{(\tilde{u}-0)\left(\tilde{u}-\frac{1}{3}\right)(\tilde{u}-1)}{\left(\frac{2}{3}-0\right)\left(\frac{2}{3}-\frac{1}{3}\right)\left(\frac{2}{3}-1\right)} \int_{0}^{1} f\left(t_{1}\right)^{-1-\alpha} d u, \quad \tilde{u} \in[0,1], \\
&\left|I_{1}\right| \leq \frac{t_{1}}{\Gamma(-\alpha)}\left(\left\|q^{(4)}\right\|_{\infty}+C_{1} h^{4}\right) \frac{1}{t_{1} \alpha}\left(t_{3 j}^{-\alpha}-\left(t_{3 j}+t_{1}\right)^{-\alpha}\right) \leq\left(\left\|p^{(4)}\right\|_{\infty}+C_{1} h^{4}\right) C_{3} t_{3 j}^{-\alpha}
\end{aligned}
$$

From (65) we have $\frac{d^{4} q(u)}{d u^{4}}=t_{1}^{4} \frac{d^{4} y(w)}{d w^{4}}$, for this reason it can be shown that

$$
\left|I_{1}\right| \leq\left(t_{1}^{4}\left\|y^{(4)}\right\|_{\infty}+C_{1} h^{4}\right) C_{3} t_{3 j}^{-\alpha} \leq C_{2} h^{4-\alpha} .
$$

Together these estimates one can end the proof

$$
\left|E_{i}(h)\right| \leq\left|\frac{1}{\mu}\right|\left(C_{2} h^{4-\alpha} C_{4} h^{4-\alpha}\right) \leq C h^{4-\alpha} .
$$


With the similar method, one can formulate the following Theorem.

Theorem 3. Set $E_{l}(h), l=3 j+2$ being the truncation error at the node $t_{i}$ defined in (60), then it holds

$$
\left|E_{i}(h)\right| \leq C h^{4-\alpha}, \quad l=3 j+2, j=1,2,3, \ldots, m-1
$$

\section{Asymptotic expansion for the error of extended algorithm}

It can be shown from subsection 2.1 in a straightforward manner that solutions of (2) satisfy,

$$
\begin{aligned}
& y\left(t_{3 j}\right)= \frac{1}{w_{0,3 j}-t_{3 j}^{\alpha} \Gamma(-\alpha) \mu}\left[t_{3 j}^{\alpha} \Gamma(-\alpha) g\left(t_{3 j}\right)-\sum_{k=1}^{3 j} w_{k, 3 j} y\left(t_{3 j-k}\right)-\frac{y_{0}}{\alpha}-R_{3 j}(f)\right], \\
& j=1,2, \ldots, m . \\
& y\left(t_{3 j+1}\right)= \frac{1}{w_{0,3 j+1}-t_{3 j+1}^{\alpha} \Gamma(-\alpha) \mu}\left[t_{3 j+1}^{\alpha} \Gamma(-\alpha) g\left(t_{3 j+1}\right)-\sum_{k=1}^{3 j} w_{k, 3 j+1} y\left(t_{3 j+1-k}\right)\right. \\
&\left.-\frac{y_{0}}{\alpha}-\int_{0}^{t_{1}}\left(t_{3 j+1}-\tau\right)^{-1-\alpha} y(\tau) d \tau-R_{3 j+1}(f)\right], \quad j=1,2, \ldots, m-1 . \\
& y\left(t_{3 j+2}\right)= \frac{1}{w_{0,3 j+2}-t_{3 j+2}^{\alpha} \Gamma(-\alpha) \mu}\left[t_{3 j+2}^{\alpha} \Gamma(-\alpha) g\left(t_{3 j+2}\right)-\sum_{k=1}^{3 j} w_{k, 3 j+2} y\left(t_{3 j+2-k}\right)\right. \\
&\left.-\frac{y_{0}}{\alpha}-\int_{0}^{t_{2}}\left(t_{3 j+2}-\tau\right)^{-1-\alpha} y(\tau) d \tau-R_{3 j+2}(f)\right], \quad j=1,2, \ldots, m-1 .
\end{aligned}
$$

In this way one can define the following numerical methods for solving (2),

$$
\begin{aligned}
y_{3 j}= & \frac{1}{w_{0,3 j}-t_{3 j}^{\alpha} \Gamma(-\alpha) \mu}\left[t_{3 j}^{\alpha} \Gamma(-\alpha) g\left(t_{3 j}\right)-\sum_{k=1}^{3 j} w_{k, 3 j} y_{3 j-k}-\frac{y_{0}}{\alpha}\right], j=1,2, \ldots, m \\
y_{3 j+1}= & \frac{1}{w_{0,3 j+1}-t_{3 j+1}^{\alpha} \Gamma(-\alpha) \mu}\left[t_{3 j+1}^{\alpha} \Gamma(-\alpha) g\left(t_{3 j+1}\right)-\sum_{k=1}^{3 j} w_{k, 3 j+1} y_{3 j+1-k}\right. \\
& \left.-\frac{y_{0}}{\alpha}-\int_{0}^{t_{1}}\left(t_{3 j+1}-\tau\right)^{-1-\alpha} y(\tau) d \tau\right], \quad j=1,2, \ldots, m-1
\end{aligned}
$$




$$
\begin{aligned}
y_{3 j+2}= & \frac{1}{w_{0,3 j+2}-t_{3 j+2}^{\alpha} \Gamma(-\alpha) \mu}\left[t_{3 j+2}^{\alpha} \Gamma(-\alpha) g\left(t_{3 j+2}\right)-\sum_{k=1}^{3 j} w_{k, 3 j+2} y_{3 j+2-k}\right. \\
& \left.-\frac{y_{0}}{\alpha}-\int_{0}^{t_{2}}\left(t_{3 j+2}-\tau\right)^{-1-\alpha} y(\tau) d \tau\right], \quad j=1,2, \ldots, m-1 .
\end{aligned}
$$

Lemma 6. Set $R_{3 j}(f), R_{3 j+1}(f)$ and $R_{3 j+2}(f)$ be the remainder terms in (7), (23) and (27), respectively. If, for some $m \geq 3, f \in C^{m+1}[0,1]$, then the sequence of remainders $R_{l}(f),(l=1,2, \ldots 3 m)$ possesses the asymptotic expansion

$$
R_{l}(f)=\sum_{\mu=4}^{m+1} d_{\mu} l^{\alpha-\mu}+\sum_{\mu=2}^{\mu^{*}} d_{\mu}^{*} l^{-2 \mu}+O\left(l^{\alpha-m-1}\right)
$$

where $\mu^{*}$ is the integer satisfying $2 \mu^{*}<m+1-\alpha<2\left(\mu^{*}+1\right)$, and $d_{\mu}$ and $d_{\mu}^{*}$ are certain coefficients that depend on $f$.

Proof. We follow the proof of Theorem 1.3 in [38] and the Lemma 2.3 in [30] where the piecewise linear and quadratic Lagrange interpolation polynomials are used respectively.

Consider the case $l=3 j, j=1,2, \ldots, m$. Let $w_{k}=\frac{k}{3 j}, k=0,1, \ldots, 3 j$ be a partition of $[0,1]$ with the step size $h_{1}=\frac{1}{3 j}$. Set $P_{3}(u)$ be the piecewise cubic Lagrange interpolation polynomial defined by (8) on $\left[w_{3 l}, w_{3 l+3}\right], l=0,1,2, \ldots, j-1$. The integration error can be represented in the form

$$
\begin{aligned}
R_{3 j}(f)= & \oint_{0}^{1} u^{-1-\alpha} f(u) d u-\oint_{0}^{1} u^{-1-\alpha} P_{3}(u) d u=\sum_{l=0}^{j-1} \int_{w_{3 l}}^{w_{3 l+3}} u^{-1-\alpha}\left(f(u)-P_{3}(u)\right) d u \\
= & \sum_{l=0}^{j-1} \int_{0}^{1}\left(w_{3 l}+3 h_{1} s\right)^{-1-\alpha}\left[f\left(w_{3 l}+3 h_{1} s\right)-\left(\left(-\frac{1}{2}(s-1)(3 s-2)(3 s-1)\right) f\left(w_{3 l}\right)\right.\right. \\
& +\frac{9}{2}(s-1) s(3 s-2) f\left(w_{3 l+1}\right)+\frac{1}{2}(-9)(s-1) s(3 s-1) f\left(w_{3 l+2}\right) \\
& \left.\left.+\frac{1}{2} s(3 s-2)(3 s-1) f\left(w_{3 l+3}\right)\right)\right]\left(3 h_{1}\right) d s .
\end{aligned}
$$

By using Taylor formula one can find

$$
\begin{aligned}
f\left(w_{3 l}\right)= & f\left(w_{3 l}+3 h_{1} s\right)+\frac{f^{\prime}\left(w_{3 l}+3 h_{1} s\right)}{1 !}\left(-3 h_{1} s\right)+\ldots+\frac{f^{(m)}\left(w_{3 l}+3 h_{1} s\right)}{m !}\left(-3 h_{1} s\right)^{m} \\
& +R_{m+1}^{1}, \\
f\left(w_{3 l+1}\right)= & f\left(w_{3 l}+3 h_{1} s\right)+\frac{f^{\prime}\left(w_{3 l}+3 h_{1} s\right)}{1 !}\left(h_{1}-3 h_{1} s\right)+\ldots+\frac{f^{(m)}\left(w_{3 l}+3 h_{1} s\right)}{m !}\left(h_{1}-3 h_{1} s\right)^{m} \\
& +R_{m+1}^{2},
\end{aligned}
$$




$$
\begin{aligned}
f\left(w_{3 l+2}\right)= & f\left(w_{3 l}+3 h_{1} s\right)+\frac{f^{\prime}\left(w_{3 l}+3 h_{1} s\right)}{1 !}\left(2 h_{1}-3 h_{1} s\right)+\ldots+\frac{f^{(m)}\left(w_{3 l}+3 h_{1} s\right)}{m !}\left(2 h_{1}-3 h_{1} s\right)^{m} \\
& +R_{m+1}^{3}, \\
f\left(w_{3 l+3}\right)= & f\left(w_{3 l}+3 h_{1} s\right)+\frac{f^{\prime}\left(w_{3 l}+3 h_{1} s\right)}{1 !}\left(3 h_{1}-3 h_{1} s\right)+\ldots+\frac{f^{(m)}\left(w_{3 l}+3 h_{1} s\right)}{m !}\left(3 h_{1}-3 h_{1} s\right)^{m} \\
& +R_{m+1}^{4},
\end{aligned}
$$

in which $R_{m+1}^{j}, j=1,2,3,4$ is the error of the Taylor approximation. For this reason one can obtain

$R_{3 j}(f)=\left(3 h_{1}\right) \sum_{l=0}^{j-1} \int_{0}^{1}\left(w_{3 l}+3 h_{1} s\right)^{-1-\alpha}\left[\sum_{r=0}^{m-4} h_{1}^{r+4} f^{(r+4)}\left(w_{3 l}+3 h_{1} s\right) \pi_{r}(s)+\varepsilon_{m+1}(s)\right] d s$,

where $\pi_{r}(s)$ are some functions of $s$ and $\varepsilon_{m+1}(s)$ depends on the reminder terms $R_{m+1}^{j}$, $j=1,2,3,4$. Following the argument of the proof for Theorem 1.3 in [38] for the latter term it is seen that

$$
\begin{aligned}
R_{3 j}(f)= & \sum_{r=0}^{m-4} h_{1}^{r+4} \int_{0}^{1}\left[\left(3 h_{1}\right) \sum_{l=0}^{j-1}\left(w_{3 l}+3 h_{1} s\right)^{-1-\alpha} f^{(r+4)}\left(w_{3 l}+3 h_{1} s\right)\right] \pi_{r}(s) d s \\
& +O\left(h_{1}^{m+1-\alpha}\right)
\end{aligned}
$$

Applying Theorem 3.2 in [39], we have,

$$
\begin{aligned}
& \left(3 h_{1}\right) \sum_{l=0}^{j-1}\left(w_{3 l}+3 h_{1} s\right)^{-1-\alpha} f^{(r+4)}\left(w_{3 l}+3 h_{1} s\right)=\frac{1}{j} \sum_{l=0}^{j-1}\left(\frac{l+s}{j}\right)^{-1-\alpha} f^{(r+4)}\left(\frac{l+s}{j}\right) \\
& =\sum_{j=0}^{m-r-3} a_{j}(s) h_{1}^{j-\alpha}+\sum_{j=0}^{m-r-4} b_{j}(s) h_{1}^{j}+O\left(h_{1}^{m-r-3}\right),
\end{aligned}
$$

with some suitable functions $a_{k}(s)$ and $b_{k}(s)$. Thus one can get

$$
\begin{aligned}
R_{3 j}(f)= & \sum_{r=0}^{m-4} \sum_{j=0}^{m-r-3}\left[\int_{0}^{1} a_{j}(s) \pi_{r}(s) d s\right] h_{1}^{4+r+j-\alpha}+\sum_{r=0}^{m-4 m-r-4} \sum_{j=0}^{1}\left[\int_{0}^{1} b_{j}(s) \pi_{r}(s) d s\right] h_{1}^{4+r+j} \\
& +O\left(h_{1}^{m+1}\right)+O\left(h_{1}^{m+1-\alpha}\right)=\sum_{\mu=4}^{m+1} d_{\mu} h_{1}^{4-\alpha}+\sum_{\mu=2}^{\mu^{*}} d_{\mu}^{*} h_{1}^{2 \mu}+O\left(h_{1}^{m+1-\alpha}\right)
\end{aligned}
$$

where $\mu^{*}$ is the integer satisfying $2 \mu^{*}<m+1-\alpha<2\left(\mu^{*}+1\right)$, and $d_{\mu}$ and $d_{\mu}^{*}$ are certain coefficients that depend on $f$ and the expansion does not contain any odd integer 
of powers of $h_{1}[38,30]$. Considering that $h_{1}=\frac{1}{3 j}$, it can be conclude that 73 holds for $l=3 j$.

For the case $l=3 j+1, j=1,2, \ldots, m-1$. Let $w_{k}=\frac{k}{3 j+1}, k=0,1, \ldots, 3 j$ be a partition of $[0,1]$ with the step size $h_{1}=\frac{1}{3 j+1}$. Then following the same argument as for the case $l=3 j$, it can be shown that we show that $(73)$ also holds for $l=3 j+1$.

For the case $l=3 j+2, j=1,2, \ldots, m-1$. Let $w_{k}=\frac{k}{3 j+2}, k=0,1, \ldots, 3 j$ be a partition of $[0,1]$ with the step size $h_{1}=\frac{1}{3 j+2}$. Then following the same argument as for the case $l=3 j$, it can be shown that we show that (73) also holds for $l=3 j+2$.

Theorem 4. Let $y\left(t_{l}\right)$ and $y_{l}$ be the exact solutions and the approximate solutions of (66) 68) respectively. Assume that the function $y \in C^{m+1}[0,1], m \geq 3$ and we obtain the exact starting values $y_{0}=y(0), y_{1}=y\left(t_{1}\right)$ and $y_{2}=y\left(t_{2}\right)$. Then there exist coefficients $c_{\mu}=c_{\mu}(\alpha)$ and $c_{\mu}^{*}=c_{\mu}^{*}(\alpha)$ such that the sequence $\left\{y_{l}\right\}, l=0,1, \ldots, 3 m$ possesses an asymptotic expansion of the form

$$
y\left(t_{3 m}\right)-y_{3 m}=\sum_{\mu=4}^{m+1} c_{\mu}(3 m)^{\alpha-\mu}+\sum_{\mu=2}^{\mu^{*}} c_{\mu}^{*}(3 m)^{-2 \mu}+O\left((3 m)^{\alpha-m-1}\right), \quad \text { for } m \rightarrow \infty,
$$

where $\mu^{*}$ is the integer satisfying $2 \mu^{*}<m+1-\alpha<2\left(\mu^{*}+1\right)$, and $d_{\mu}$ and $d_{\mu}^{*}$ are certain coefficients that depend on $y$.

Proof. We follow the proof of Theorem 2.1 in [38] and the proof of Theorem 2.1 in [30]. Fix $t_{l}=c, l=1,2, \ldots, 3 m$ to be a constant. The following difference will be investigated:

$$
e_{l}=y\left(t_{l}\right)-y_{l}, \quad \text { for } l \rightarrow \infty, \text { with } t_{l}=l h=\frac{l}{3 m}=c
$$

where $h=1 /(3 m)$ is the step size. In other words, there is a constant $c$, independent of $m$, such that

$$
l=c \cdot(3 m), \quad \text { or } \quad m=l /(3 c),
$$

and consequently, one can see that if $e_{l}$ possesses an asymptotic expansion with respect to $l$, then $e_{3 m}$ possesses at the same time one with respect to $m$, and vice versa. We shall 
prove

$$
e_{l}=\sum_{\mu=4}^{m+1} c_{\mu}(3 m)^{\alpha-\mu}+\sum_{\mu=2}^{\mu^{*}} c_{\mu}^{*}(3 m)^{-2 \mu}+O\left((3 m)^{\alpha-m-1}\right), \quad \text { for } l \rightarrow \infty,
$$

125 Consider the case $l=3 j, j=1,2, \ldots, m$. Utilizing the fact that $t_{3 j}=3 j \cdot h=\frac{3 j}{3 m}=c$, it follows from Eqs. (66) and (69) that

$$
e_{3 j}=\frac{1}{c^{\alpha} \Gamma(-\alpha) \mu-w_{0,3 j}}\left[\sum_{k=1}^{3 j} w_{k, 3 j} e_{3 j-k}+R_{3 j}(f)\right]
$$

Recall that $f(\cdot)=y\left(t_{3 j}-t_{3 j}\right) \in C^{m+1}[0,1], m \geq 3$, for this reason we have, by Lemma 6

$$
R_{3 j}(f)=\sum_{\mu=4}^{m+1} d_{\mu}(3 j)^{\alpha-\mu}+\sum_{\mu=2}^{\mu^{*}} d_{\mu}^{*}(3 j)^{-2 \mu}+O\left((3 j)^{\alpha-m-1}\right) \quad \text { for } j \rightarrow \infty .
$$

Using the fact that $(3 j) /(3 m)=c$ one can find

$$
R_{3 j}(f)=\sum_{\mu=4}^{m+1} \tilde{d}_{\mu}(3 m)^{\alpha-\mu}+\sum_{\mu=2}^{\mu^{*}} \tilde{d}_{\mu}^{*}(3 m)^{-2 \mu}+O\left((3 m)^{\alpha-m-1}\right) \quad \text { for } j \rightarrow \infty .
$$

The principle of mathematical induction will be used to prove that (74) holds for the coefficients $c_{\mu}=\frac{1}{-c^{\alpha} \Gamma(-\alpha) \mu-1 / \alpha} \tilde{d}_{\mu}$ and $c_{\mu}^{*}=\frac{1}{-c^{\alpha} \Gamma(-\alpha) \mu-1 / \alpha} \tilde{d}_{\mu}^{*}$. By assumption $e_{0}=$ $e_{1}=e_{2}=0$, hence (74) holds for $l=0,1,2$. Consider the case 3. Applying Lemma 6 leads to

$$
\begin{aligned}
e_{3} & =\frac{1}{c^{\alpha} \Gamma(-\alpha) \mu-w_{0,3}}\left[\sum_{k=1}^{3} w_{k, 3} e_{3-k}+R_{3}(f)\right]=\frac{1}{c^{\alpha} \Gamma(-\alpha) \mu-w_{0,3}}[ \\
& \left.\left(\sum_{k=0}^{3} w_{k, 3}-w_{0,3}\right)\left(\sum_{\mu=4}^{m+1} c_{\mu}(3 m)^{\alpha-\mu}+\sum_{\mu=2}^{\mu^{*}} c_{\mu}^{*}(3 m)^{-2 \mu}+O\left((3 m)^{\alpha-m-1}\right)\right)+R_{3}(f)\right]
\end{aligned}
$$

Using the fact that $w_{0, l}=\frac{\left(2 \alpha^{2}+8 \alpha+12\right)(3 m c)^{\alpha}}{3^{\alpha} 2(-\alpha)(-\alpha+1)(-\alpha+2)(-\alpha+3)}$ and $\sum_{k=0}^{3} w_{k, 3}=-1 / \alpha$ it follows 
that

$$
\begin{aligned}
& \quad\left[\frac{\left(2 \alpha^{2}+8 \alpha+12\right)(3 m c)^{\alpha}}{3^{\alpha} 2(-\alpha)(-\alpha+1)(-\alpha+2)(-\alpha+3)}-c^{\alpha} \Gamma(-\alpha) \mu\right] e_{3}= \\
& \frac{1}{\alpha}\left[\sum_{\mu=4}^{m+1} c_{\mu}(3 m)^{\alpha-\mu}+\sum_{\mu=2}^{\mu^{*}} c_{\mu}^{*}(3 m)^{-2 \mu}+O\left((3 m)^{\alpha-m-1}\right)\right] \\
& -\left[\sum_{\mu=4}^{m+1} \tilde{d}_{\mu}(3 m)^{\alpha-\mu}+\sum_{\mu=2}^{\mu^{*}} \tilde{d}_{\mu}^{*}(3 m)^{-2 \mu}+O\left((3 m)^{\alpha-m-1}\right)\right] \\
& +\frac{\left(2 \alpha^{2}+8 \alpha+12\right)(3 m c)^{\alpha}}{3^{\alpha} 2(-\alpha)(-\alpha+1)(-\alpha+2)(-\alpha+3)}\left[\sum_{\mu=4}^{m+1} c_{\mu}(3 m)^{\alpha-\mu}+\sum_{\mu=2}^{\mu^{*}} c_{\mu}^{*}(3 m)^{-2 \mu}\right. \\
& \left.\quad+O\left((3 m)^{\alpha-m-1}\right)\right]
\end{aligned}
$$

This shows that the sequence $e_{2}$ possesses an asymptotic expansion with respect to the powers of $3 m$, and it is easy to check that (74) hold for $l=3[38,30]$. Suppose that Eq. (74) is true for $l=0,1, \ldots, 3 j-1$. Following the same argument for (76) and using the fact that $\sum_{k=0}^{3 j} w_{k, 3 j}=-1 / \alpha$ it follows that

$$
\begin{aligned}
& {\left[\frac{\left(2 \alpha^{2}+8 \alpha+12\right)(3 m c)^{\alpha}}{3^{\alpha} 2(-\alpha)(-\alpha+1)(-\alpha+2)(-\alpha+3)}-c^{\alpha} \Gamma(-\alpha) \mu\right] e_{3 j}=} \\
& \frac{1}{\alpha}\left[\sum_{\mu=4}^{m+1} c_{\mu}(3 m)^{\alpha-\mu}+\sum_{\mu=2}^{\mu^{*}} c_{\mu}^{*}(3 m)^{-2 \mu}+O\left((3 m)^{\alpha-m-1}\right)\right] \\
& -\left[\sum_{\mu=4}^{m+1} \tilde{d}_{\mu}(3 m)^{\alpha-\mu}+\sum_{\mu=2}^{\mu^{*}} \tilde{d}_{\mu}^{*}(3 m)^{-2 \mu}+O\left((3 m)^{\alpha-m-1}\right)\right] \\
& +\frac{\left(2 \alpha^{2}+8 \alpha+12\right)(3 m c)^{\alpha}}{3^{\alpha} 2(-\alpha)(-\alpha+1)(-\alpha+2)(-\alpha+3)}\left[\sum_{\mu=4}^{m+1} c_{\mu}(3 m)^{\alpha-\mu}+\sum_{\mu=2}^{\mu^{*}} c_{\mu}^{*}(3 m)^{-2 \mu}\right. \\
& \left.\quad+O\left((3 m)^{\alpha-m-1}\right)\right]
\end{aligned}
$$

This shows that the sequence $e_{3 j}$ possesses an asymptotic expansion with respect to the powers of $3 m$, and it is easy to check that (74) hold for $l=3 j[38,30]$.

By a completely similar argument it can be shown that (74) is hold for $l=3 j+1$ ${ }_{130} \quad$ and $l=3 j+2$. 


\section{Numerical results}

To check the numerical errors between the exact and the numerical solution and to verify the convergence order of the numerical algorithm with respect to the step size $h$ numerical experiments are carried out in this section. The experimental orders of convergence (EOC) is measured by the following ratios [23]

$$
E O C=\log _{2}\left(\frac{E(h)}{E(h / 2)}\right)
$$

where $E(h)$ is the absolute error $\left|y\left(t_{l}\right)-y_{l}\right|$ with step size $h$. As demonstrated by the above section, the order of convergence of the algorithm is $O\left(h^{4-\alpha}\right)$. The idea in [30] is pursued to investigate the graphical representations for the convergence order.

$$
|E(h)|=\left|y\left(t_{l}\right)-y_{l}\right| \leq C h^{4-\alpha} \Rightarrow \log _{2}(|E(h)|)=\log _{2}(C)+(4-\alpha) \log _{2}(h),
$$

to observe the order of convergence we will plot the strait line $y=(4-\alpha) x$ and also $y=y(x)$ where $y=\log _{2}(|E(h)|)$ and $x=\log _{2}(h)$. Parallelism of this two lines means that the order of convergence of the numerical algorithm is $O\left(h^{4-\alpha}\right)$.

Example 1. Consider the following fractional differential equation

$$
{ }_{0}^{C} D_{t}^{\alpha} y(t)=\frac{24}{\Gamma(5-\alpha)} t^{4-\alpha}-\frac{3}{\Gamma(4-\alpha)} t^{3-\alpha}-\frac{1}{2} t^{3}-y(t)+t^{4} ; \quad y(0)=0 .
$$

The exact solution is $y(t)=t^{4}-\frac{1}{2} t^{3}$. Tables (1) and (2) show the absolute errors at the time $t=1$ between the exact solution and the numerical solution of the presented algorithm with different $\alpha \in(0,1)$. From these Tables, it is observed that the experimental order of convergence of the numerical results is of order $O\left(h^{4-\alpha}\right)$ for the presented algorithm as expected to the results of the previous section. Tables (1) and (2) show the value of the absolute error reduction as the grid size $h$ is decreased.

The order of convergence for various choices of $\alpha$ is plotted in Fig. (2). This figure contains the strait line $y=(4-\alpha) x$ and also $y=y(x)$ where $y=\log _{2}(|E(h)|)$ and $x=\log _{2}(h)$. One can see that these two lines are exactly parallel for all cases which confirms the order of convergence of the numerical method. The compare of Tables (11) ${ }_{45}$ and(4) and Fig. (2) show the effectiveness of the novel algorithm and indicates that the numerical results are consistent with the theoretical ones. 
Example 2. Consider the following fractional differential equation

$$
{ }_{0}^{C} D_{t}^{\alpha} y(t)=\mu y(t)+g(t), \quad y(0)=0
$$

where

$$
\mu=-1, \quad g(t)=\frac{\Gamma(4+\gamma)}{\Gamma(4+\gamma-\alpha)} t^{3+\gamma-\alpha}-\mu t^{3+\gamma}, \quad \gamma>0 .
$$

The exact solution is $y(t)=t^{3+\gamma}$. At the time $t=1$ for different step sizes $h$ and different $\alpha$, the approximate solutions for the given equation are obtained for two values of $\gamma$. The absolute errors and the experimental order of convergence of the novel algorithm (59) for $\gamma-0.7,1.7$ are shown in Tables (3)-(6). These Tables show that novel scheme is valid methods in solving the fractional differential equation.

Figs. (3) and (4) depict the order of convergence for various choices of $\alpha$ and $\gamma$. One can observe that the two lines in these Figs. are exactly parallel which means that the order of convergence of the novel method is $O\left(h^{4-\alpha}\right)$. This is well in line with the prediction of the previous section. The compare of Tables (3)-(6) and Figs. (3) and (4) indicates that the novel algorithm is effective and also the experimental orders of convergence support the theoretical convergent orders.

\section{Conclusion}

This paper provides a novel algorithm with theoretically proved convergence order of $O\left(h^{4-\alpha}\right)$ for solving fractional differential equations. The algorithm is based on a discretisation of the Hadamard finite-part integral, which is used to express the Riemann-Liouville fractional derivative. The cubic Lagrange polynomials are utilized to approximate the integral. Numerical estimation of the starting integrals and the starting nodes with acceptable accuracy is presented. The detailed error analysis is discussed to establish the high order accuracy of the method. The numerical examples are provided to show the effectiveness and convergence orders of our numerical algorithms. It is shown that the numerical results are consistent with the theoretical ones. The quite similar procedure can be applied to design an algorithm for FDEs with order $1<\alpha<2$. In the future, we shall try to follow this idea to construct a higher 
order scheme for solving nonlinear FDEs, as well as to apply the presented algorithm to fractional partial differential equations.

\section{Appendix}

The idea of solving $y_{1}, y_{2}$ and $y_{3}$ form (59) is as follows.

$$
\left\{\begin{array}{c}
y_{1}=\frac{1}{M_{1}}\left(t_{1}^{\alpha} \Gamma(-\alpha) g\left(t_{1}\right)-\tilde{\tilde{w}}_{0,1} y_{0}-\tilde{\tilde{w}}_{2,1} y_{2}-\tilde{\tilde{w}}_{3,1} y_{3}\right) \\
y_{2}=\frac{1}{M_{2}}\left(t_{2}^{\alpha} \Gamma(-\alpha) g\left(t_{2}\right)-\tilde{\tilde{w}}_{0,2} y_{0}-\tilde{\tilde{w}}_{1,2} y_{1}-\tilde{\tilde{w}}_{3,2} y_{3}\right) \\
y_{3}=\frac{1}{M_{3}}\left(t_{3}^{\alpha} \Gamma(-\alpha) g\left(t_{3}\right)-\tilde{\tilde{w}}_{1,3} y_{2}-\tilde{\tilde{w}}_{2,3} y_{1}-\tilde{\tilde{w}}_{3,3} y_{0}\right)
\end{array}\right.
$$

where

$$
M_{1}=w_{1,1}-t_{1}^{\alpha} \Gamma(-\alpha) \mu, \quad M_{2}=w_{2,2}-t_{2}^{\alpha} \Gamma(-\alpha) \mu, \quad M_{3}=w_{0,3}-t_{3}^{\alpha} \Gamma(-\alpha) \mu
$$

Putting Eq. (82) into Eq. (82) yields

$$
\begin{aligned}
y_{2}= & \frac{1}{1-\frac{\tilde{\tilde{w}}_{3,2} \tilde{\tilde{w}}_{1,3}}{M_{2} M_{3}}}\left[\frac{t_{2}^{\alpha} \Gamma(-\alpha)}{M_{2}} g\left(t_{2}\right)-\frac{\tilde{\tilde{w}}_{3,2} t_{3}^{\alpha} \Gamma(-\alpha)}{M_{2} M_{3}} g\left(t_{3}\right)+\left(\frac{-\tilde{\tilde{w}}_{0,2}}{M_{2}}+\frac{\tilde{\tilde{w}}_{3,2} \tilde{w}_{3,3}}{M_{2} M_{3}}\right) y_{0}\right. \\
& \left.-\left(\frac{-\tilde{\tilde{w}}_{1,2}}{M_{2}}+\frac{\tilde{w}_{3,2} \tilde{w}_{2,3}}{M_{2} M_{3}}\right) y_{1}\right] .
\end{aligned}
$$

Substituting (83) and (82) to (82) leads to

$$
\begin{aligned}
y_{1}= & \frac{1}{1-\frac{Y_{1}^{1}}{Y_{1}^{0}}}\left[\frac{t_{1}^{\alpha} \Gamma(-\alpha)}{M_{1}} g\left(t_{1}\right)+\frac{t_{2}^{\alpha} \Gamma(-\alpha)\left(-\tilde{\tilde{w}}_{3,1} \tilde{\tilde{w}}_{1,3}+\tilde{\tilde{w}}_{2,1} M_{3}\right)}{M_{1}\left(\tilde{\tilde{w}}_{3,2} \tilde{\tilde{w}}_{1,3}-M_{2} M_{3}\right)} g\left(t_{2}\right)\right. \\
& \left.+\frac{t_{3}^{\alpha} \Gamma(-\alpha)\left(-\tilde{\tilde{w}}_{2,1} \tilde{\tilde{w}}_{3,2}+\tilde{\tilde{w}}_{3,1} M_{2}\right)}{M_{1}\left(\tilde{\tilde{w}}_{3,2} \tilde{w}_{1,3}-M_{2} M_{3}\right)} g\left(t_{3}\right)+\frac{Y_{1}^{2}}{Y_{1}^{0}} y_{0}\right]
\end{aligned}
$$

where

$Y_{1}^{0}=\tilde{\tilde{w}}_{3,2} \tilde{\tilde{w}}_{1,3} M_{1}-M_{1} M_{2} M_{3}, \quad Y_{1}^{1}=\tilde{\tilde{w}}_{1,2} \tilde{\tilde{w}}_{3,1} \tilde{\tilde{w}}_{1,3}+\tilde{\tilde{w}}_{2,1} \tilde{\tilde{w}}_{3,2} \tilde{\tilde{w}}_{2,3}-\tilde{\tilde{w}}_{3,1} \tilde{\tilde{w}}_{2,3} M_{2}-\tilde{\tilde{w}}_{1,2} \tilde{\tilde{w}}_{2,1} M_{3}$, $Y_{1}^{2}=\tilde{\tilde{w}}_{0,2} \tilde{\tilde{w}}_{3,1} \tilde{\tilde{w}}_{1,3}-\tilde{\tilde{w}}_{0,1} \tilde{\tilde{w}}_{3,2} \tilde{\tilde{w}}_{1,3}+\tilde{\tilde{w}}_{2,1} \tilde{\tilde{w}}_{3,2} \tilde{\tilde{w}}_{3,3}-\tilde{\tilde{w}}_{3,1} \tilde{\tilde{w}}_{3,3} M_{2}-\tilde{\tilde{w}}_{0,2} \tilde{\tilde{w}}_{2,1} M_{3}+\tilde{\tilde{w}}_{0,1} M_{2} M_{3}$.

In this way, firstly one can calculate $y_{1}$ from given initial conditions and the known function $g(t)$. Then $y_{2}$ and $y_{3}$ can be calculated by (83) and (82), respectively. 
[1] S. David, J. Linares, E. Pallone, Fractional order calculus: historical apologia, basic concepts and some applications, Revista Brasileira de Ensino de Física 33 (4) (2011) 4302-4302.

[2] L. Chen, Y. Chai, R. Wu, J. Yang, Stability and stabilization of a class of nonlinear fractional-order systems with caputo derivative, IEEE Transactions on Circuits and Systems II: Express Briefs 59 (9) (2012) 602-606.

[3] F. Padula, A. Visioli, et al., Advances in robust fractional control, Springer, 2015.

[4] D. Wang, A. Xiao, H. Liu, Dissipativity and stability analysis for fractional functional differential equations, Fractional Calculus and Applied Analysis 18 (6) (2015) 1399-1422.

[5] M. P. Aghababa, A. R. Haghighi, M. Roohi, Stabilisation of unknown fractionalorder chaotic systems: an adaptive switching control strategy with application to power systems, IET Generation, Transmission \& Distribution 9 (14) (2015) 1883-1893.

[6] S. T. Mohyud-Din, T. Nawaz, E. Azhar, M. A. Akbar, fractional sub-equation method to spacetime fractional calogero-degasperis and potential kadomtsevpetviashvili equations, Journal of Taibah University for Science 11 (2) (2017) $258-263$.

[7] A. Nagy, N. Sweilam, An efficient method for solving fractional hodgkin-huxley model, Physics Letters A 378 (30) (2014) 1980-1984.

[8] B. Ahmad, S. K. Ntouyas, A. Alsaedi, On a coupled system of fractional differential equations with coupled nonlocal and integral boundary condiitons, Chaos, Solitons \& Fractals 83 (2016) 234-241.

[9] M. S. Asl, M. Javidi, An improved pc scheme for nonlinear fractional differential equations: error and stability analysis, Journal of Computational and Applied Mathematics (2017) (Accepted manuscript). 
[10] S. Ji, G. Li, Solutions to nonlocal fractional differential equations using a noncompact semigroup, Electronic Journal of Differential Equations 2013 (240) (2013) 1-14.

[18] A. Arafa, S. Rida, M. Khalil, A fractional-order model of hiv infection with drug therapy effect, Journal of the Egyptian Mathematical Society 22 (3) (2014) 538543. 
[19] V. Daftardar-Gejji, Y. Sukale, S. Bhalekar, A new predictor-corrector method for fractional differential equations, Applied Mathematics and Computation 244 (2014) 158-182.

[20] M. S. Kushwaha, A. Kumar, et al., An approximate solution to a moving boundary problem with space-time fractional derivative in fluvio-deltaic sedimentation process, Ain Shams Engineering Journal 4 (4) (2013) 889-895.

[21] N. J. Ford, A. C. Simpson, The numerical solution of fractional differential equations: speed versus accuracy, Numerical Algorithms 26 (4) (2001) 333-346.

[22] Z. Feng, N. Chen, On the existence and uniqueness of the solution of linear fractional differential-algebraic system, Mathematical Problems in Engineering 2016.

[23] W. Deng, C. Li, Numerical schemes for fractional ordinary differential equations, in: P. Miidla (Ed.), Numerical Modelling, InTech, Rijeka, 2012, Ch. 16, pp. 355374.

[24] K. Diethelm, N. J. Ford, A. D. Freed, A predictor-corrector approach for the numerical solution of fractional differential equations, Nonlinear Dynamics 29 (14) (2002) 3-22.

[25] K. Diethelm, N. J. Ford, A. D. Freed, Detailed error analysis for a fractional adams method, Numerical algorithms 36 (1) (2004) 31-52.

[26] C. Li, Q. Yi, A. Chen, Finite difference methods with non-uniform meshes for nonlinear fractional differential equations, Journal of Computational Physics 316 (2016) 614-631.

250 [27] K. Diethelm, An algorithm for the numerical solution of differential equations of fractional order, Electronic transactions on numerical analysis 5 (1) (1997) 1-6.

[28] N. Ford, M. Manuela Rodrigues, N. Vieira, A numerical method for the fractional schrödinger type equation of spatial dimension two, Fractional Calculus and Applied Analysis 16 (2) (2013) 454-468. 
[29] N. J. Ford, J. Xiao, Y. Yan, Stability of a numerical method for a space-timefractional telegraph equation, Computational Methods in Applied Mathematics Comput. Methods Appl. Math. 12 (3) (2012) 273-288.

[30] Y. Yan, K. Pal, N. J. Ford, Higher order numerical methods for solving fractional differential equations, BIT Numerical Mathematics 54 (2) (2014) 555-584.

[31] N. J. Ford, K. Pal, Y. Yan, An algorithm for the numerical solution of two-sided space-fractional partial differential equations., Comput. Meth. in Appl. Math. 15 (4) (2015) 497-514.

[32] K. Pal, F. Liu, Y. Yan, Numerical solutions of fractional differential equations by extrapolation, in: I. Dimov, I. Faragó, L. Vulkov (Eds.), Finite Difference Methods, Theory and Applications, Springer International Publishing, 2015, pp. 299-306.

[33] Z. Li, Y. Yan, N. J. Ford, Error estimates of a high order numerical method for solving linear fractional differential equations, Applied Numerical Mathematics 114 (2017) 201-220.

[34] D. ELLIOTT, An asymptotic analysis of two algorithms for certain hadamard finite-part integrals, IMA journal of numerical analysis 13 (3) (1993) 445-462.

[35] I. Podlubny, Fractional differential equations: an introduction to fractional derivatives, fractional differential equations, to methods of their solution and some of their applications, Vol. 198, Academic press, 1998.

[36] K. DIETTELM, Generalized compound quadrature formulae for finite-part integrals, IMA journal of numerical analysis 17 (3) (1997) 479-493.

[37] J. Cao, C. Xu, A high order schema for the numerical solution of the fractional ordinary differential equations, Journal of Computational Physics 238 (2013) 154168.

[38] K. Diethelm, G. Walz, Numerical solution of fractional order differential equations by extrapolation, Numerical algorithms 16 (3) (1997) 231-253. 
[39] J. Lyness, Finite-part integrals and the euler-maclaurin expansion, in: R. Zahar (Ed.), Approximation and Computation, Birkhäuser Boston, 1994, pp. 397-407.

Table 1: The absolute errors and the convergence orders of the numerical algorithm [59] for 78].

\begin{tabular}{cccccc} 
& \multicolumn{2}{c}{$\alpha=0.4$} & & \multicolumn{2}{c}{$\alpha=0.6$} \\
\cline { 2 - 3 } \cline { 5 - 6 }$h=1 /(3 m)$ & $E(h)$ & $E O C$ & & $E(h)$ & $E O C$ \\
\hline $1 / 15$ & $4.5157 \mathrm{e}-05$ & - & & $1.4314 \mathrm{e}-04$ & - \\
$1 / 30$ & $3.8319 \mathrm{e}-06$ & 3.56 & & $1.3975 \mathrm{e}-05$ & 3.36 \\
$1 / 60$ & $3.2131 \mathrm{e}-07$ & 3.58 & & $1.3430 \mathrm{e}-06$ & 3.38 \\
$1 / 120$ & $2.6782 \mathrm{e}-08$ & 3.58 & & $1.2816 \mathrm{e}-07$ & 3.39 \\
$1 / 240$ & $2.2304 \mathrm{e}-09$ & 3.59 & & $1.2195 \mathrm{e}-08$ & 3.39 \\
\hline
\end{tabular}

Table 2: The absolute errors and the convergence orders of the numerical algorithm [59] for 78].

\begin{tabular}{cccccc}
\hline & \multicolumn{2}{c}{$\alpha=0.8$} & & \multicolumn{2}{c}{$\alpha=0.9$} \\
\cline { 2 - 3 } \cline { 5 - 6 }$h=1 /(3 m)$ & $E(h)$ & $E O C$ & & $E(h)$ & $E O C$ \\
\hline $1 / 15$ & $4.0049 \mathrm{e}-04$ & - & & $6.4792 \mathrm{e}-04$ & - \\
$1 / 30$ & $4.5293 \mathrm{e}-05$ & 3.14 & & $7.8956 \mathrm{e}-05$ & 3.04 \\
$1 / 60$ & $5.0120 \mathrm{e}-06$ & 3.18 & & $9.3830 \mathrm{e}-06$ & 3.07 \\
$1 / 120$ & $5.4971 \mathrm{e}-07$ & 3.19 & & $1.1039 \mathrm{e}-06$ & 3.09 \\
$1 / 240$ & $6.0043 \mathrm{e}-08$ & 3.20 & & $1.2929 \mathrm{e}-07$ & 3.10 \\
\hline
\end{tabular}


Table 3: The absolute errors and the convergence orders of the numerical algorithm 59 for 79 with $\gamma=0.7$.

\begin{tabular}{cccccc}
\hline & \multicolumn{2}{c}{$\alpha=0.4$} & & \multicolumn{2}{c}{$\alpha=0.6$} \\
\cline { 2 - 3 } \cline { 6 - 6 }$h=1 /(3 m)$ & $E(h)$ & $E O C$ & & $E(h)$ & $E O C$ \\
\hline $1 / 15$ & $2.5332 \mathrm{e}-05$ & - & & $8.3210 \mathrm{e}-05$ & - \\
$1 / 30$ & $2.1379 \mathrm{e}-06$ & 3.5667 & & $8.1151 \mathrm{e}-06$ & 3.3581 \\
$1 / 60$ & $1.7885 \mathrm{e}-07$ & 3.5794 & & $7.8038 \mathrm{e}-07$ & 3.3784 \\
$1 / 120$ & $1.4893 \mathrm{e}-08$ & 3.5860 & & $7.4561 \mathrm{e}-08$ & 3.3877 \\
$1 / 240$ & $1.2160 \mathrm{e}-09$ & 3.6144 & & $7.0085 \mathrm{e}-09$ & 3.4112 \\
\hline
\end{tabular}

Table 4: The absolute errors and the convergence orders of the numerical algorithm 59 for 79 with $\gamma=0.7$.

\begin{tabular}{ccccccc}
\hline & \multicolumn{3}{c}{$\alpha=0.8$} & & \multicolumn{2}{c}{$\alpha=0.9$} \\
\cline { 2 - 3 } \cline { 6 - 6 }$h=1 /(3 m)$ & $E(h)$ & $E O C$ & & $E(h)$ & $E O C$ \\
\hline $1 / 15$ & $2.4220 \mathrm{e}-04$ & - & & $4.0023 \mathrm{e}-04$ & - \\
$1 / 30$ & $2.7493 \mathrm{e}-05$ & 3.14 & & $4.9075 \mathrm{e}-05$ & 3.03 \\
$1 / 60$ & $3.0536 \mathrm{e}-06$ & 3.17 & & $5.8617 \mathrm{e}-06$ & 3.07 \\
$1 / 120$ & $3.3596 \mathrm{e}-07$ & 3.18 & & $6.9235 \mathrm{e}-07$ & 3.08 \\
$1 / 240$ & $3.6853 \mathrm{e}-08$ & 3.19 & & $8.1320 \mathrm{e}-08$ & 3.09 \\
\hline
\end{tabular}

Table 5: The absolute errors and the convergence orders of the numerical algorithm 59 for 79 with $\gamma=1.7$.

\begin{tabular}{cccccc}
\hline & \multicolumn{2}{c}{$\alpha=0.4$} & & \multicolumn{2}{c}{$\alpha=0.6$} \\
\cline { 2 - 3 } \cline { 6 - 6 }$h=1 /(3 m)$ & $E(h)$ & $E O C$ & & $E(h)$ & $E O C$ \\
\hline $1 / 15$ & $1.2073 \mathrm{e}-04$ & - & & $3.5787 \mathrm{e}-04$ & - \\
$1 / 30$ & $1.0519 \mathrm{e}-05$ & 3.52 & & $3.5742 \mathrm{e}-05$ & 3.32 \\
$1 / 60$ & $8.9480 \mathrm{e}-07$ & 3.56 & & $3.4764 \mathrm{e}-06$ & 3.36 \\
$1 / 120$ & $7.5186 \mathrm{e}-08$ & 3.57 & & $3.3390 \mathrm{e}-07$ & 3.38 \\
$1 / 240$ & $6.2667 \mathrm{e}-09$ & 3.58 & & $3.1822 \mathrm{e}-08$ & 3.39 \\
\hline
\end{tabular}


Table 6: The absolute errors and the convergence orders of the numerical algorithm 59] for 79] with $\gamma=1.7$.

\begin{tabular}{cccccc}
\hline & \multicolumn{2}{c}{$\alpha=0.8$} & & \multicolumn{2}{c}{$\alpha=0.9$} \\
\cline { 2 - 3 } \cline { 5 - 6 }$h=1 /(3 m)$ & $E(h)$ & $E O C$ & & $E(h)$ & $E O C$ \\
\hline $1 / 15$ & $9.2807 \mathrm{e}-04$ & - & & $1.4415 \mathrm{e}-03$ & - \\
$1 / 30$ & $1.0690 \mathrm{e}-04$ & 3.12 & & $1.7852 \mathrm{e}-04$ & 2.97 \\
$1 / 60$ & $1.1948 \mathrm{e}-05$ & 3.16 & & $2.1410 \mathrm{e}-05$ & 3.06 \\
$1 / 120$ & $1.3175 \mathrm{e}-06$ & 3.18 & & $2.5313 \mathrm{e}-06$ & 3.08 \\
$1 / 240$ & $1.4434 \mathrm{e}-07$ & 3.19 & & $2.9722 \mathrm{e}-07$ & 3.09 \\
\hline
\end{tabular}

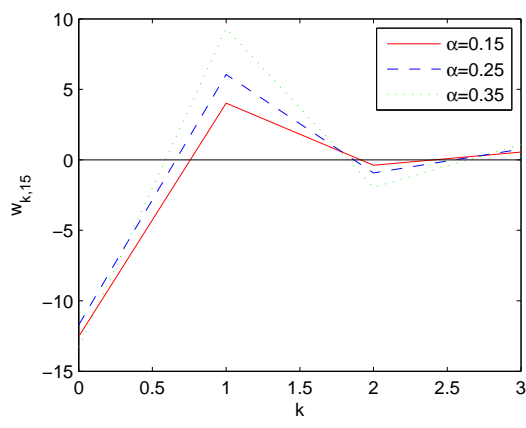

(a)

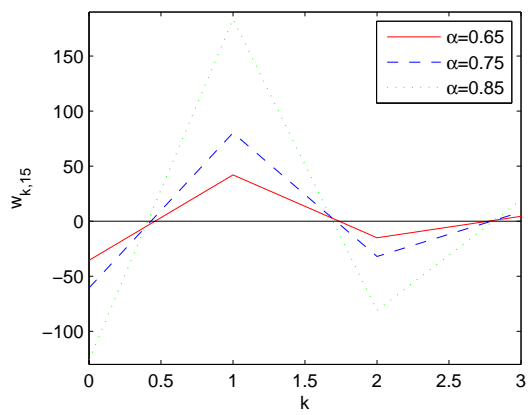

(c)

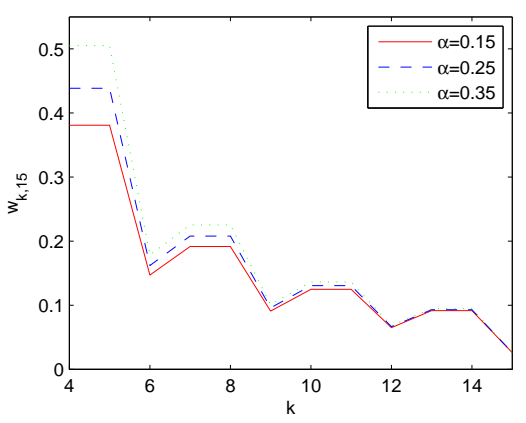

(b)

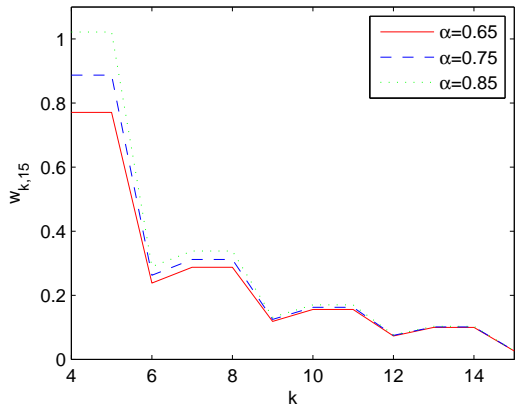

(d)

Figure 1: The weights $w_{k, 15}$ with the different fractional order $\alpha$. 


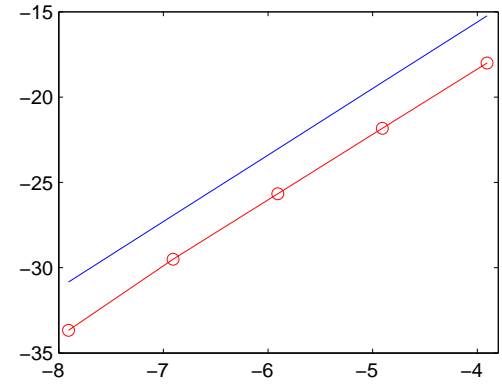

(a) $\alpha=0.1$

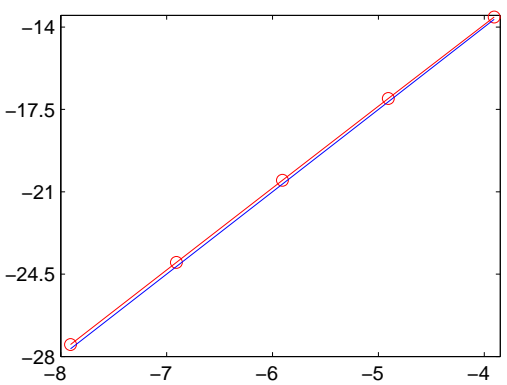

(c) $\alpha=0.5$

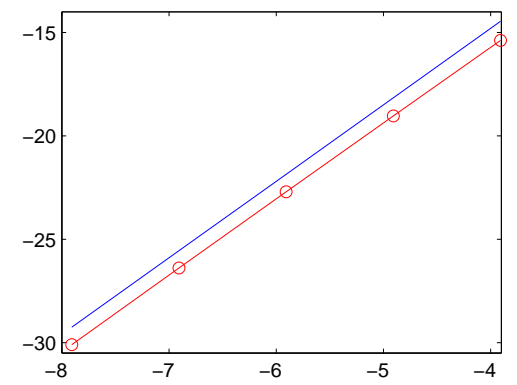

(b) $\alpha=0.3$

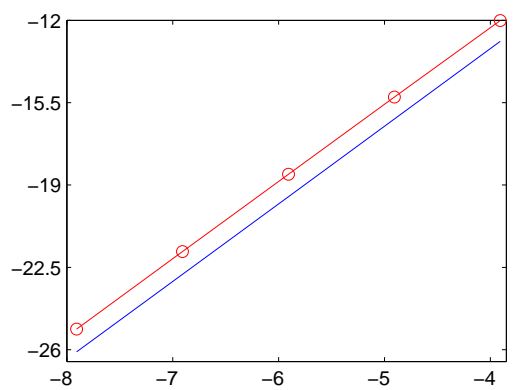

(d) $\alpha=0.7$

Figure 2: The experimentally determined orders of convergence for 78 . 


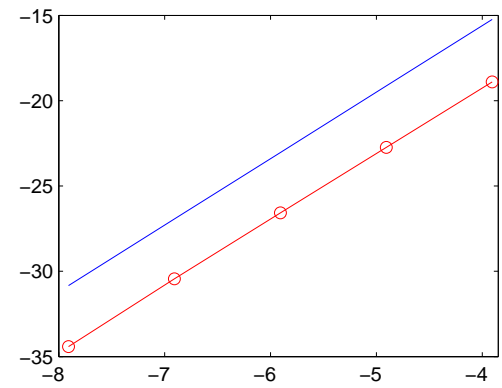

(a) $\alpha=0.1$

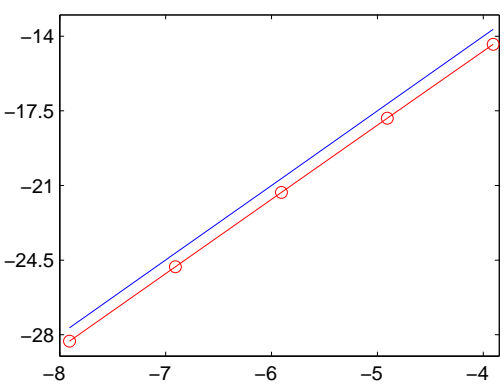

(c) $\alpha=0.5$

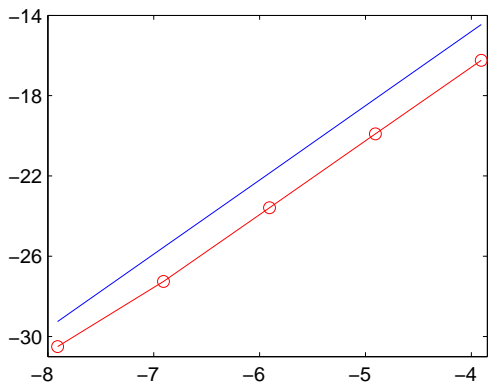

(b) $\alpha=0.3$

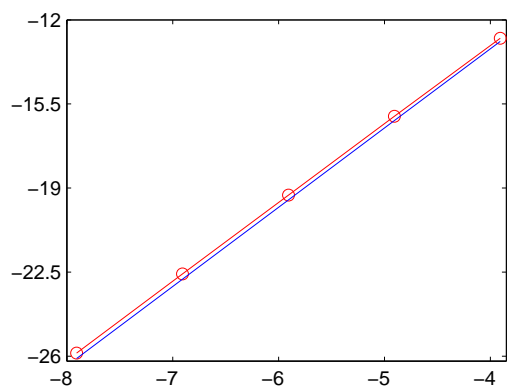

(d) $\alpha=0.7$

Figure 3: The experimentally determined orders of convergence for 79 with $\gamma=0.7$. 


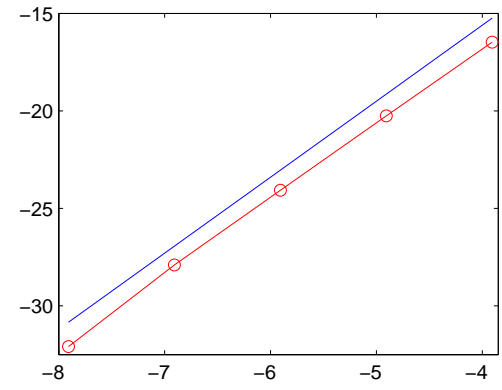

(a) $\alpha=0.1$

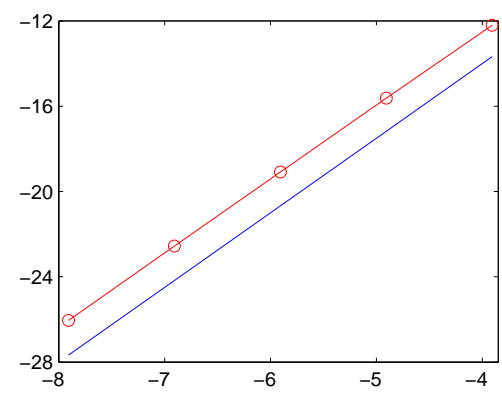

(c) $\alpha=0.5$

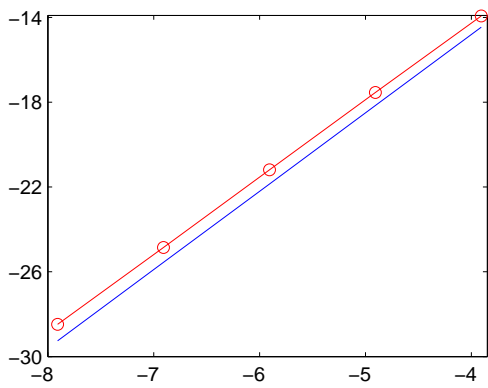

(b) $\alpha=0.3$

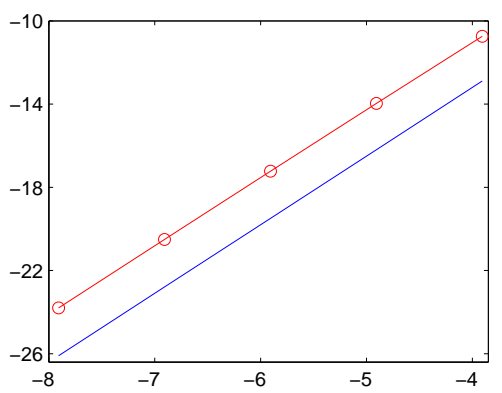

(d) $\alpha=0.7$

Figure 4: The experimentally determined orders of convergence for 79 with $\gamma=0.7$. 